\title{
COUNTING OPEN NODAL LINES OF RANDOM WAVES ON PLANAR DOMAINS
}

\author{
JOHN A. TOTH AND IGOR WIGMAN
}

\begin{abstract}
We compute the asymptotic expectation of the number of open nodal lines for random waves on smooth planar domains. We find that for both the long energy window $[0, \lambda]$, and the short one $[\lambda, \lambda+1]$, the expected number of open nodal lines is proportional to $\lambda$, asymptotically as $\lambda \rightarrow \infty$. Our results are consistent with the predictions of Blum, Gnutzmann and Smilansky [BGS] in the physics literature.
\end{abstract}

\section{INTRODUCTION}

Let $\Omega \subset \mathbb{R}^{2}$ be a smooth planar domain and consider the Dirichlet problem

$$
-\Delta \varphi_{j}=\lambda_{j}^{2} \varphi_{j} ;\left.\quad \varphi_{j}\right|_{\partial \Omega}=0 .
$$

Here, we assume that $\int_{\Omega} \varphi_{i} \varphi_{j} d x=\delta_{j}^{i}$. Consider the zero set of the $j$-th Dirichlet eigenfunction (with the boundary excluded)

$$
\mathcal{N}_{j}:=\left\{x \in \Omega ; \varphi_{j}(x)=0\right\}-\partial \Omega .
$$

We call the set $\mathcal{N}_{j}$ the nodal set of the eigenfunction $\phi_{j}$. This set is a curve which in general has self intersections. However, for generic domains [U], a nodal set is a union of connected components consisting of closed loops homeomorphic to circles and open nodal lines homeomorphic to open intervals. In this paper, we compute the average number of open nodal lines for a random linear combination of Dirichlet eigenfunctions in various spectral intervals. This problem is generically equivalent to counting the zeros of the boundary traces of the eigenfunctions. The latter problem has obvious similarities to measuring the length of the interior nodal line, and our results show that the order of magnitude is the same. We recall that S. T. Yau conjectured that in all dimensions, the hypersurface volume should satisfy $c \lambda_{j} \leq \mathcal{H}^{n-1}\left(\mathcal{N}_{j}\right) \leq C \lambda_{j}$ for some positive constants $c, C$ depending only on $(M, g)$ [Y1, Y 2 . The lower bound was proved in dimension two for smooth domains by Brüning-Gomes [BG] and both the upper and lower bounds were proved in all dimensions for analytic $(M, g)$ by Donnelly-Fefferman [DF, DF2]. Recently, there have been important advances in understanding other aspects of the geometry of nodal sets for large eigenvalues, including asymptotic results for the expected number of nodal domains for random spherical harmonics [NS], as well as for the distribution of nodal sets on general Riemannian manifolds without boundary [Z2].

The first author was partially supported by NSERC grant \# OG0170280 and a William Dawson Fellowship

The second author is supported by a CRM-ISM Fellowship. 
To state our results, we define the random linear combination of eigenfunctions (i.e. random waves) corresponding to the long range energy window

$$
\Phi_{a}^{L}(x ; \lambda):=\sum_{\lambda_{j} \in[0, \lambda]} a_{j} \varphi_{j}(x),
$$

and the random combination corresponding to the short range energy window

$$
\Phi_{a}^{S}(x ; \lambda)=\sum_{\lambda_{j} \in[\lambda, \lambda+1]} a_{j} \varphi_{j}(x),
$$

where in both cases, $a_{j}$ are $(0,1)$ Gaussian i.i.d defined on the sample space $\left(\mathbb{R}^{N^{L, S}(\lambda)} ; e^{-\|a\|^{2} / 2} \frac{d a}{(2 \pi)^{N^{L, S}(\lambda) / 2}}\right)$ and

$$
N^{L}(\lambda):=\#\left\{\lambda_{k} \in[0, \lambda]\right\} ; \quad N^{S}(\lambda):=\#\left\{\lambda_{k} \in[\lambda, \lambda+1]\right\} .
$$

The nodal set of the random wave $\Phi_{a}^{L, S}$ is by definition the curve

$$
\mathcal{N}_{a}^{L, S}(\lambda)=\left\{x \in \Omega ; \Phi_{a}^{L, S}(x ; \lambda)=0\right\}-\partial \Omega .
$$

We are interested here in computing the asymptotics of the number of intersections of the nodal set with the boundary, $\partial \Omega$. Let

$$
\mathcal{I}_{a}^{L, S}(\lambda)=\operatorname{card}\left(\overline{\mathcal{N}}_{a}^{L, S}(\lambda) \cap \partial \Omega\right) .
$$

Since for generic domains, a nodal set is a union of closed loops homeomorphic to circles and open nodal lines homeomorphic to open intervals, the intersection number $\mathcal{I}_{a}^{L, S}(\lambda)$, is therefore, almost surely, equal to twice the number of open nodal lines of the nodal set, $\mathcal{N}_{a}^{L, S}(\lambda)$; with probability 1 , there are no multiple intersections with the boundary (see Lemma 3.1).

Given the random variables, $\mathcal{I}_{a}^{L, S}(\lambda)$, we define the corresponding expectations

$$
\mathcal{Z}^{L, S}(\lambda)=\mathbb{E}_{a}^{L, S}(\lambda) .
$$

Our main result is the computation of the leading asymptotics of $\mathcal{Z}^{L, S}(\lambda)$ as $\lambda \rightarrow \infty$. For the following theorem, we need to impose a generic nonrecurrence condition. We say that a point $q \in \partial \Omega$ is non-recurrent if the measure of loops at $q \in \partial \Omega$ for the associated billiard map $\beta: B^{*} \partial \Omega \rightarrow B^{*} \partial \Omega$ is zero. In the following, we denote the arclength of $\partial \Omega$ by $\ell(\partial \Omega)$.

Theorem 1.1. Let $\varphi_{j} ; j=1,2, \ldots$ be the Dirichlet eigenfunctions of a smooth domain $\Omega$ and asssume that all boundary points are non-recurrent. Then, given $a_{j} ; j=1, \ldots, N^{L, S}(\lambda)$, i.i.d. $(0,1)$-Gaussian random variables,

$$
\text { (i) } \mathcal{Z}^{L}(\lambda)=\frac{\ell(\partial \Omega)}{\sqrt{6} \pi} \lambda+o(\lambda),
$$

and

$$
\text { (ii) } \mathcal{Z}^{S}(\lambda)=\frac{\ell(\partial \Omega)}{2 \pi} \lambda+o(\lambda)
$$

as $\lambda \rightarrow \infty$.

Remark 1.2. The non-recurrence assumption in Theorem 1.1 holds for generic boundaries and, in particular, for all convex analytic domains [Z1]. 
The result in Theorem 1.1 is related to well-known results of Berard $[\mathrm{Be}]$ on the expected nodal lengths of random superpositions of eigenfunctions and Berry [BR], who computed the expected length of nodal lines for isotropic, monochromatic random waves in the plane (eigenfunctions of the free Laplacian). He also gave a somewhat heuristic argument for computing the asymptotics of the variance. Of more direct relevance is the recent result by Zelditch [Z2] on the expected nodal distribution of random waves on compact manifolds without boundary. One can naturally view Theorem 1.1 as the analogue for domains with boundary. Blum, Gnutzmann and Smilansky [BGS] $]^{1}$ have studied the distribution of the number of boundary intersections $\tilde{\nu_{j}}$ of the nodal set of $\varphi_{j}$ (in addition to the number of nodal domains). Using Berry's random wave to model $\phi_{j}$ for chaotic systems, they found that for large eigenvalues, $\tilde{\nu}_{j}$ should concentrate around $\frac{\ell}{2 \pi} \cdot \lambda_{j}$, where $\ell$ is the length of the boundary $\partial \Omega$. Numerical results for eigenfunctions of both Sinai and stadium billiards support this prediction. Since for general domains, the average nodal asymptotics over spectral intervals $[\lambda, \lambda+1]$ should be the same as for individual eigenfunctions of ergodic billiards, our asymptotic result for $\mathcal{Z}^{S}(\lambda)$ in Theorem 1.1 is thus consistent with [BGS].

Recently, in the case of piecewise-analytic domains, Toth and Zelditch [TZ1] have proved deterministic upper bounds for the number of nodal intersections with the boundary $\partial \Omega$ (and more general interior curves) for individual eigenfunctions. In work in progress, when $\Omega$ is an ergodic billiard, these authors have also proved some asymptotic results for the nodal (and critical point) distributions of complexified restrictions of Dirichlet and Neumann eigenfunctions along strictly convex, real-analytic interior curves $C \subset \Omega$. In this case, at least for an ergodic sequence of eigenfunctions, the number of complex zeros of the holomorphic continuations of the eigenfunction restrictions, $\left.\phi_{j}\right|_{C}$, is $\sim c \lambda$. The same result is likely true when $C=\partial \Omega$ and this would of course be consistent with the random result in Theorem 1.1 .

In examples like the torus or sphere, where the spectrum of $\Omega$ is degenerate with high multiplicity, rather than summing eigenfunctions belonging to different eigenspaces, it is natural to consider the ensemble of random eigenfunctions attached to fixed eigenspaces. A natural way to do so is to fix a basis $B=\left\{\eta_{1}, \ldots \eta_{\mathcal{N}}\right\}$ of the eigenspace $\mathcal{E}_{\lambda}$ and consider the random ensemble of functions on $\Omega$ defined by

$$
\eta=a_{1} \eta_{1}+\ldots+a_{\mathcal{N}} \eta_{\mathcal{N}}
$$

where $a_{i}$ are standard Gaussian i.i.d. Note that the probability density of $\eta$ is independent of the choice of the basis $B$.

Berard [Be] computed the expected length for the nodal line of a random eigenfunction on the sphere to be const $\cdot \lambda$. Rudnick and Wigman [RW] and Wigman [W] have studied the variance of the length of the nodal line of random eigenfunctions with $\lambda \rightarrow \infty$, for the torus and the sphere respectively.

Recently, Granville and Wigman [GW] have determined the asymptotics of the variance of number of zeros of random trigonometric polynomials of

\footnotetext{
${ }^{1}$ We thank Zeev Rudnick for pointing out this reference
} 
degree $\sim \lambda$ and moreover, proved a central limit theorem for their distribution. While there are clearly similarities between the boundary traces of (2) and (3) and random trigonometric polynomials of degree $\sim \lambda$, it would likely be difficult to prove a central limit theorem for nodal distributions of random waves on arbitrary smooth domains. However, we do hope to study the variance and higher moments in future work.

We thank Zeev Rudnick for many helpful discussions about random zeros, Steve Zelditch for helpful comments regarding pointwise Weyl laws at the boundary and the anonymous referee for helpful comments and suggestions. The second author would also like to thank the CRM analysis laboratory and its members for their support.

\section{A PRELIMINARY LEMMA ON NODAL INTERSECTIONS WITH $\partial \Omega$}

As above, we let $\Omega$ be a smooth bounded domain in $\mathbb{R}^{2}$ and let $\varphi_{j}$ be the $L^{2}$ normalized eigenfunction of the Laplacian with eigenvalue $\lambda_{j}^{2}$ satisfying the Dirichlet boundary conditions. Without loss of generality, we assume that the eigenfunctions are real-valued and we let

$$
q:[0, \ell] \rightarrow \partial \Omega, \theta \mapsto q(\theta)=\left(q_{1}(\theta), q_{2}(\theta)\right)
$$

be the arclength parametrization of the boundary $\partial \Omega$ with $\ell:=\ell(\partial \Omega)$.

Let $v_{j}:[0, \ell] \rightarrow \mathbb{R}$ be the boundary trace of $\varphi_{j}$, that is,

$$
v_{j}(\theta):=\partial_{\nu} \varphi_{j}(q(\theta)) \text {. }
$$

Here, $\nu=\nu(q)$ denotes the unit outward-pointing normal to the boundary. Recall that the main object of our interest are the real valued random variables $\Phi_{a}^{L}(x ; \lambda)$ and $\Phi_{a}^{S}(x ; \lambda)$ (see (2) and (3)).

We would like to compute the leading asymptotics of the expectation $\mathcal{Z}^{L, S}(\lambda)=\mathbb{E} \mathcal{I}_{a}^{L, S}(\lambda)$ and we do this by counting the zeros of the boundary traces of $\Phi_{a}^{L}$ and $\Phi_{a}^{S}$ defined by

$$
V_{a}^{L}(\theta ; \lambda)=\sum_{\lambda_{j} \in[0, \lambda]} a_{j} v_{j}(\theta)
$$

and

$$
V_{a}^{S}(\theta ; \lambda)=\sum_{\lambda_{j} \in[\lambda, \lambda+1]} a_{j} v_{j}(\theta) .
$$

The functions $V_{a}^{L, S}(\cdot ; \lambda)$ are useful, since, as we show in the next section, their zeros correspond to the intersections of the nodal sets $\mathcal{N}_{a}^{L, S}(\lambda)$ with the boundary $\partial \Omega$, for a generic choice of $a$. In particular, we will show that the number $\mathcal{I}_{a}^{L, S}(\lambda)$ of the intersections of the nodal line $\mathcal{N}_{a}^{L, S}(\lambda)$ with the boundary equals the number of the zeros of $V_{a}^{L, S}(\theta ; \lambda)$, almost surely (see Lemma 2.1). This observation implies that

$$
\mathcal{Z}^{L, S}(\lambda):=\mathbb{E}\left[\mathcal{I}_{a}^{L, S}(\lambda)\right]=\mathbb{E}\left[\tilde{\mathcal{I}}_{a}^{L, S}(\lambda)\right],
$$

where

$$
\tilde{\mathcal{I}}_{a}^{L, S}(\lambda)=\#\left\{\theta \in[0, \ell]: V_{a}^{L, S}(\theta ; \lambda)=0\right\} .
$$

Our main interest here is in the asymptotic behaviour of $\mathcal{Z}^{L, S}(\lambda)$, as $\lambda \rightarrow$ $\infty$. In both cases we reduce the problem of counting the nodal intersections to counting the number of zeros of random functions, for which we use the 
Kac-Rice formula. There are related formulas that have been used by several authors in different settings: In a series of papers starting from [SZ such formulas are used to study the distribution of zeros of random holomorphic sections of vector bundles. A more classical reference for such formulas is CL].

2.1. Open nodal lines and boundary critical points. In the Dirichlet case treated here, the key to computing the $\lambda \rightarrow \infty$ asymptotics of $\mathcal{Z}(\lambda)$ is the observation that generically the number of boundary critical points of $\Phi_{a}(\theta ; \lambda)$ equals twice the number of open nodal lines. The following elementary result makes this correspondence more precise.

We begin with the following result for functions vanishing on $\partial \Omega$. It is an elementary result which is probably known (see e.g. [BGS]), but since we could not find a direct reference, we include the proof here.

Lemma 2.1. Let $\Phi \in C^{\infty}(\Omega)$ satisfy $\Phi(q)=0$ for all $q \in \partial \Omega$. Assume that the critical points of $\Phi$ on $\partial \Omega$ are simple. Then the number of open nodal lines of $\Phi$ intersecting $\partial \Omega$ equals $\frac{1}{2}$ the number of boundary critical points.

Proof. Let $\mathcal{N}_{\Phi}$ be the nodal set of $\Phi$. First, we assume that $q$ is an intersection of $\mathcal{N}_{\Phi}$ with $\partial \Omega$. To show that $\partial_{\nu} \Phi(q)=0$, we flatten out the boundary and introduce local normal coordinates $\left(x_{1}, x_{2}\right) \in(-\epsilon, \epsilon)^{2}$ with $x(q)=(0,0)$ and also, $x_{2}(p)=0$ for all $p \in \partial \Omega$ with $x_{2}>0$ in $\Omega$.

We locally have either

$$
\left(f\left(x_{2}\right), x_{2}\right) \in \mathcal{N}_{\Phi}
$$

for some $f \in C^{\infty}(-\epsilon, \epsilon)$ with $f(0)=0$, or alternatively,

$$
\left(x_{1}, g\left(x_{1}\right)\right) \in \mathcal{N}_{\Phi}
$$

for a $C^{\infty}(-\epsilon, \epsilon)$ function $g$ with $g(0)=g^{\prime}(0)=0$, where $g$ doesn't vanish identically on any interval containing 0 (the latter case is if the nodal line is tangent to the boundary; only the former case is possible, see the proof of the converse statement below). In fact, the simple zeros assumption ensures that locally there is an equality rather than an inclusion (see the proof of the converse statement).

In case (9), the Taylor expansion of $\Phi\left(x_{1}, x_{2}\right)$ around $\left(x_{1}, 0\right)$ gives

$$
\Phi\left(f\left(x_{2}\right), x_{2}\right)=\partial_{x_{2}} \Phi\left(f\left(x_{2}\right), 0\right) x_{2}+\mathcal{O}\left(x_{2}^{2}\right)=0,
$$

since $\Phi\left(x_{1}, 0\right)=0$, so that

$$
\partial_{x_{2}} \Phi\left(f\left(x_{2}\right), 0\right)=\mathcal{O}\left(x_{2}\right) .
$$

Since $f(0)=0$, it follows that $\partial_{x_{2}} \Phi(0,0)=0$, which finishes the proof in that case.

In case (10), similar reasoning gives

$$
\partial_{x_{2}} \Phi\left(x_{1}, 0\right)=\mathcal{O}\left(g\left(x_{1}\right)\right),
$$

provided that $g\left(x_{1}\right)$ does not vanish identically. Since $g(0)=0$, it follows again that $\partial_{x_{2}} \Phi(0,0)=0$.

To prove the converse, assume that $q \in \partial \Omega$ is a critical point of $\Phi$ and show that $q$ is a nodal intersection point with the boundary, $\partial \Omega$. Using the 
same normal coordinates as in the previous proof, we consider the equation

$$
\Phi\left(x_{1}, x_{2}\right)=0,
$$

where, by assumption $\partial_{x_{2}} \Phi(0,0)=0$. The fact that $\Phi\left(x_{1}, 0\right)=0$ implies that

$$
\Phi\left(x_{1}, x_{2}\right)=x_{2} \psi\left(x_{1}, x_{2}\right)
$$

with $\psi(0,0)=0$ and $\partial_{x_{1}} \psi(0,0) \neq 0$. It follows by the implicit function theorem that $\psi\left(x_{1}, x_{2}\right)=0$ is locally the graph $x_{2}=g\left(x_{1}\right)$.

\section{A KAC-Rice FORMUla For $\mathcal{Z}^{L, S}(\lambda)$}

3.1. Some notation. Given a point $\theta \in[0, \ell]$ and the spectral parameter $\lambda \in \mathbb{R}^{+}$, we introduce the vectors $b_{1}^{L, S}(\theta ; \lambda), b_{2}^{L, S}(\theta ; \lambda) \in \mathbb{R}^{N^{L, S}(\lambda)}$ where

$$
b_{1}^{L}(\theta ; \lambda)=\left(v_{k}(\theta)\right)_{\lambda_{k} \in[0, \lambda]} ; \quad b_{2}^{L}(\theta ; \lambda)=\left(\partial_{\theta} v_{k}(\theta)\right)_{\lambda_{k} \in[0, \lambda]}
$$

for the long spectral range, and

$$
b_{1}^{S}(\theta ; \lambda)=\left(v_{k}(\theta)\right)_{\lambda_{k} \in[\lambda, \lambda+1]} ; \quad b_{2}^{S}(\theta ; \lambda)=\left(\partial_{\theta} v_{k}(\theta)\right)_{\lambda_{k} \in[\lambda, \lambda+1]}
$$

for the short range. Here the dimension is given by (4). Note that for $\lambda$ sufficiently large, for every $\theta \in[0, \ell]$, the vectors $b_{1}^{L, S}(\theta ; \lambda)$ and $b_{2}^{L, S}(\theta ; \lambda)$ are not collinear (see Corollary 6.1). We will use this fact in the proof of the main result of this section (Proposition 3.2).

In addition to the vectors $b^{L, S}(\theta ; \lambda)$ of boundary traces of eigenfunctions it is also useful to define the corresponding functions $c_{i j}^{L, S}(\theta ; \lambda):[0, \ell] \rightarrow \mathbb{R}$ for $i, j \in\{1,2\}$, where

$$
c_{i j}^{L}(\theta ; \lambda):=\left\langle b_{i}^{L}(\theta ; \lambda), b_{j}^{L}(\theta ; \lambda)\right\rangle ; \quad c_{i j}^{S}(\theta ; \lambda):=\left\langle b_{i}^{S}(\theta ; \lambda), b_{j}^{S}(\theta ; \lambda)\right\rangle .
$$

For example, $c_{11}^{L, S}$ is just the squared length of $b_{1}^{L, S}$ and $c_{12}^{L, S}=c_{21}^{L, S}$.

3.2. A Kac-Rice formula. In this section we give a Kac-Rice type formula for computing the expected value of the number of zeros of a random combination of Dirichlet eigenfunctions. Results similar to the one here can be found in [CL] (see pg. 285). Bleher, Shiffman and Zelditch BSZ1, BSZ2] have proved a Kac-Rice formula which applies in a more general situation. However, for the convenience of the reader, we give a direct elementary proof of Proposition 3.2 in the appendix.

The following Lemma 3.1 is used in the proof of Proposition 3.2. It implies that with probability 1 all the zeros of the boundary trace are simple and together with Lemma2.1 this implies the equality (8), i.e. that the expected number of nodal intersection with the boundary equals the expected number of zeros of the boundary trace. The proof of Lemma 3.1 is also given in the appendix.

Lemma 3.1. For $\lambda$ sufficiently large, the set

$$
\mathcal{C}=\left\{a \in \mathbb{R}^{N^{L, S}(\lambda)}: \exists \theta \in[0, \ell] . V_{a}^{L, S}(\theta ; \lambda)=\partial_{\theta} V_{a}^{L, S}(\theta ; \lambda)=0\right\}
$$

satisfies $\mu(\mathcal{C})=0$ where $d \mu(a):=(2 \pi)^{-N^{L, S}(\lambda) / 2} e^{-\|a\|^{2} / 2} d a$. Moreover,

$$
\operatorname{codim} \mathcal{C} \geq 1 \text {. }
$$


Proposition 3.2. The expected number of nodal intersections with the boundary $\partial \Omega$ is given by

$$
\mathcal{Z}^{L, S}(\lambda)=\frac{1}{\pi} \int_{0}^{\ell} \sqrt{\frac{c_{22}^{L, S}(\theta ; \lambda)}{c_{11}^{L, S}(\theta ; \lambda)}-\left(\frac{c_{12}^{L, S}(\theta ; \lambda)}{c_{11}^{L, S}(\theta ; \lambda)}\right)^{2}} d \theta,
$$

with $c_{i j}^{L, S}(\theta ; \lambda)$ defined in section 3.1 .

In the following sections 4 and 5 , we show that the leading term on the RHS of (14) is $\sim C_{\Omega}^{L, S} \lambda$ for some universal constants $C_{\Omega}^{L, S}>0$.

\section{Asymptotics for $\mathcal{Z}^{L}(\lambda)$.}

In this section, we compute the large- $\lambda$ asymptotics of the pointwise sums $c_{11}^{L}(\theta ; \lambda)=\sum_{\lambda_{j} \leq \lambda}\left|v_{j}(\theta)\right|^{2}$ and $c_{22}^{L}(\theta ; \lambda)=\sum_{\lambda_{j} \leq \lambda}\left|\partial_{\theta} v_{j}(\theta)\right|^{2}$. The leading asymptotics for the special case of $c_{11}^{L}(\theta ; \lambda)$ was computed by Ozawa $\mathrm{Oz}$ and the asymptotics for the pointwise sum $c_{22}^{L}(\theta ; \lambda)$ is closely-related, but we could not find it in the literature. For the benefit of the reader, we give the computation of both of the diagonal sums here. Alternatively, the leading asymptotics for $c_{11}^{L}(\theta ; \lambda)$ and $c_{22}^{L}(\theta ; \lambda)$ follow from wave analysis in section 5. However, since it is more elementary to use the heat calculus for manifolds with boundary (and also provides an independent verification of the asymptotics), this is the approach we take in this section. The required bound for the mixed sum $c_{12}^{L}(\theta ; \lambda)$ is more subtle and requires the full twoterm asymptotics arising from the wave analysis in section 5 (see (49)).

The relevant heat parametrix construction goes back to work of R. Seeley [S] and here we briefly recall the main results that will be needed for our computations. The reader can find more detailed treatments in [HZ Appendix 12, $[\mathrm{S}$ and $[\mathrm{Oz}$. Roughly speaking, the Seeley parametrix for the Dirichlet (or Neumann) resolvent for an elliptic boundary value problem is constructed as a sum of an interior parametrix and a Poisson-kernel-type correction which compensates for the boundary conditions. To describe it in more detail, it is useful to introduce normal coordinates $(x, y) \in \mathbb{R}^{2}$ in a neighbourhood of the boundary so that the Euclidean metric takes the form $d x^{2}+h(x, y) d y^{2}$ and the domain is given in these local coordinates by $x \geq 0$, the boundary corresponding to $x=0$. Consequently, since $q:[0, \ell] \rightarrow \partial \Omega$ is the arclength parametrization,

$$
d \theta^{2}=h(0, y) d y^{2} .
$$

We let $(x, y) \in U$ where $U$ is a sufficiently thin tubular neighbourhood of the boundary, $\partial \Omega$.

In local coordinates, the $N$-th order Seeley parametrix for the Dirichlet resolvent $R_{\Omega}(\mu):=\left(-\Delta_{\Omega}-\mu\right)^{-1}$ (here, $\left.\mu=\lambda^{2}\right)$ is of the form

$$
\begin{aligned}
& R_{\Omega, N}(\mu)=\sum_{j=0}^{N}(2 \pi)^{-2} \int_{\mathbb{R}^{2}} \int_{\mathbb{R}^{2}} e^{i\left(x-x^{\prime}\right) \xi} e^{i\left(y-y^{\prime}\right) \eta} c_{-2-j}(x, y, \xi, \eta, \mu) h^{-1 / 2}(x, y) d \xi d \eta \\
& +\sum_{j=0}^{N}(2 \pi)^{-2} \int_{\mathbb{R}^{2}} \int_{\mathbb{R}^{2}} e^{-i x^{\prime} \xi} e^{i\left(y-y^{\prime}\right) \eta} d_{-2-j}(x, y, \xi, \eta, \mu) h^{-1 / 2}(x, y) d \xi d \eta
\end{aligned}
$$


In (16) the factor $h^{-1 / 2}(x, y)$ is included in the integrand to cancel the coefficient in the volume measure $h^{1 / 2}(x, y)|d x d y|$ upon integration. The first term on the RHS in (16) is the interior parametrix and the second one is the boundary-compensating parametrix. Here, we are mainly interested in the leading coefficients, $c_{-2}, d_{-2}$. A direct computaton in local coordinates shows that

$$
c_{-2}(x, y, \xi, \eta, \lambda)=\left(\xi^{2}+|\eta|_{h}^{2}-\mu\right)^{-1}
$$

where,

$$
|\eta|_{h}^{2}:=h^{-1}(x, y) \eta^{2} .
$$

For the subsequent terms in the asymptotic series $\sum_{j=0}^{N} c_{-2-j}$,

$$
\sup _{x, y \in U}\left|D_{x, y}^{\alpha} c_{-2-j}(x, y, \xi, \eta ;-i \mu)\right| \leq C_{\alpha}\left(\left.|\xi||+| \eta|+| \mu\right|^{1 / 2}\right)^{-2-j} ; j \geq 1,|\alpha| \geq 0 .
$$

Here, we abuse notation somewhat and denote the holomorphic continuation of $c_{-2-j}$ to the cone $\Sigma=\left\{(\zeta, \mu) \in \mathbb{C}^{2} \times \mathbb{C} ; \Im \mu \leq\left(|\Re \mu|+|\Re \zeta|^{2}-|\Im \zeta|^{2}\right)\right\}$ with $\zeta(\xi, \eta)$ also by $c_{-2-j}$. For the boundary-compensating terms one has

$$
d_{-2}(x, y, \xi, \eta, \mu)=-\frac{e^{-x \sqrt{|\xi|_{h}^{2}-\mu}}}{\xi^{2}+|\eta|_{h}^{2}-\mu}, \quad \Re \sqrt{|\eta|_{h}^{2}-\mu}>0, \quad x \geq 0 .
$$

In this case, the subsequent terms in the sum $\sum_{j=0}^{N} d_{-2-j}$ satisfy estimates of the form

$$
\begin{gathered}
\sup _{x, y \in U}\left|x^{\alpha} D_{x}^{\beta} d_{-2-j}(x, y, \xi, \eta,-i \mu)\right| \leq C_{\alpha, \beta}\left(|\xi|+|\eta|+|\mu|^{1 / 2}\right)^{-1-j-|\alpha|+|\beta|} \\
\times \exp \left(-\delta_{\alpha, \beta} y\left(|\xi|+|\eta|+|\mu|^{1 / 2}\right)\right) ; \quad \delta_{\alpha, \beta} \geq 0 .
\end{gathered}
$$

To get a parametrix for the Dirichlet heat kernel of $\Omega$, one writes the heat kernel as a contour integral

$$
e^{-t \Delta_{\Omega}}=\frac{i}{2 \pi} \int_{\Gamma} e^{-t \mu}\left(-\Delta_{\Omega}-\mu\right)^{-1} d \mu .
$$

Here, $\Gamma=\left\{-L+s e^{ \pm i \theta}, s \geq 0\right\}$ with $L>0$ and $\theta \in\left(0, \frac{\pi}{2}\right)$, is a wedge enclosing the spectrum of $\Delta_{\Omega}$.

Substitution of the resolvent parametrix (16) in the contour integral (18) gives the following expression for the heat kernel parametrix in $U \times U$ :

$$
\begin{gathered}
H_{N}(t)\left(x, y ; x^{\prime}, y^{\prime}\right)=\sum_{j=0}^{N}(2 \pi)^{-2} \iint e^{i\left(x-x^{\prime}\right) \xi} e^{i\left(y-y^{\prime}\right) \eta} \gamma_{j}(x, y, \xi, \eta, t) h^{-1 / 2}(x, y) d \xi d \eta \\
+\sum_{j=0}^{N}(2 \pi)^{-2} \iint e^{-i x^{\prime} \xi} e^{i\left(y-y^{\prime}\right) \eta} \delta_{j}(x, y, \xi, \eta, t) h^{-1 / 2}(x, y) d \xi d \eta .
\end{gathered}
$$

It is well-known that $H_{N}(t)$ is a good approximation to $e^{-t \Delta_{\Omega}}$ in the sense that for $t \geq 0$,

$$
\left|\partial_{z}^{\alpha} \partial_{w}^{\beta}\left(e^{-t \Delta_{\Omega}}(z, w)-H_{N}(t)(z, w)\right)\right| \leq C_{\alpha, \beta} t^{[-(n+\alpha+\beta)+N+1] / 2} e^{-\delta|z-w|^{2} / t} ; \delta>0
$$


where $n=\operatorname{dim} \Omega=2$. By the Cauchy integral formula,

$\gamma_{0}(x, y, \xi, \eta, t)=\frac{i}{2 \pi} \int_{\Gamma} e^{-t \mu} c_{-2}(x, y, \xi, \eta, \mu) d \mu=\frac{i}{2 \pi} \int_{\Gamma} \frac{e^{-t \mu}}{\xi^{2}+|\eta|_{h}^{2}-\mu} d \mu=e^{-t\left(\xi^{2}+|\eta|_{h}^{2}\right)}$.

Similarily,

$$
\begin{gathered}
\delta_{0}(x, y, \xi, \eta, t)=\frac{i}{2 \pi} \int_{\Gamma} e^{-t \mu} d_{-2}(x, y, \xi, \eta, \mu) d \mu=-\frac{i}{2 \pi} \int_{\Gamma} e^{-t \mu} \frac{e^{-x \sqrt{|\eta|_{h}^{2}-\mu}}}{\xi^{2}+|\eta|_{h}^{2}-\mu} d \mu \\
=-e^{-t\left(\xi^{2}+|\eta|_{h}^{2}\right)} \times e^{-i x \xi} .
\end{gathered}
$$

So, subsititution of the formulas for $\gamma_{0}$ and $\delta_{0}$ and differentiation under the integral sign in (19) gives

$$
\sum_{j} e^{-t \lambda_{j}^{2}}\left|v_{j}(\theta(y))\right|^{2}=\left.\partial_{x} \partial_{x^{\prime}} H(t)\left(x, y ; x^{\prime}, y^{\prime}\right)\right|_{x=x^{\prime}=0, y=y^{\prime}}+\mathcal{O}\left(t^{-\frac{3}{2}}\right)
$$$$
=\left.(2 \pi)^{-2} h^{-1 / / 2}(0, y) \iint e^{-t \xi^{2}} \partial_{x} \partial_{x^{\prime}}\left[e^{-t|\eta|_{h}^{2}} e^{-i x^{\prime} \xi}\left(e^{i x \xi}-e^{-i x \xi}\right)\right]\right|_{x=x^{\prime}=0} d \xi d \eta+\mathcal{O}\left(t^{-\frac{3}{2}}\right)
$$

$=\left.(2 \pi)^{-2} h^{-1 / 2}(0, y) \iint e^{-t \xi^{2}}(-i \xi) \partial_{x}\left[e^{-t|\eta|_{h}^{2}}\left(e^{i x \xi}-e^{-i x \xi}\right)\right]\right|_{x=0} d \xi d \eta+\mathcal{O}\left(t^{-\frac{3}{2}}\right)$

In the last line, differentiation of $e^{-t|\eta|_{h}^{2}}$ gives an integrand that is odd in $\xi$ and so it integrates to zero. Thus,

$$
\begin{gathered}
\sum_{j} e^{-t \lambda_{j}^{2}}\left|\partial_{\nu} \phi_{j}(0, y)\right|^{2}=2(2 \pi)^{-2} h^{-1 / 2}(0, y) \int e^{-t \xi^{2}} \xi^{2} d \xi \times \int e^{-t|\eta|_{h}^{2}(0, y)} d \eta+\mathcal{O}\left(t^{-\frac{3}{2}}\right) \\
=a(y) t^{-2}+\mathcal{O}\left(t^{-\frac{3}{2}}\right)
\end{gathered}
$$

where $a(y)=\frac{1}{4 \pi}$. By the Karamata Tauberian theorem,

$$
c_{11}^{L}(\theta ; \lambda)=\sum_{j ; \lambda_{j}^{2} \leq \mu}\left|v_{j}(\theta)\right|^{2} \sim_{\lambda \rightarrow \infty} c_{11}^{L}(\theta) \mu^{2}=c_{11}^{L}(\theta) \lambda^{4},
$$

where,

$$
c_{11}^{L}(\theta)=(8 \pi)^{-1} .
$$

Similarily, the asymptotics for the second pointwise Weyl sum $\sum_{\lambda_{j}^{2} \leq \mu}\left|\partial_{\theta} v_{j}(\theta(y))\right|^{2}$ follows from the formula

$\sum_{\lambda_{j}^{2} \leq \mu} e^{-t \lambda_{j}^{2}}\left|\partial_{\theta} v_{j}(\theta)\right|^{2}=\left.(2 \pi)^{-2} h^{-1 / 2}(0, y)\left|\partial_{\theta} y\right|^{2} \cdot\left[\partial_{y} \partial_{y^{\prime}} \partial_{x} \partial_{x^{\prime}} H(t)\left(x, y ; x^{\prime}, y^{\prime}\right)\right]\right|_{x=x=0, y=y^{\prime}}$

$=2(2 \pi)^{-2} h^{-1 / 2}(0, y)\left|\partial_{\theta} y\right|^{2} \iint e^{-t \xi^{2}} e^{-t h^{-1}(0, y) \eta^{2}} \xi^{2} \eta^{2} d \xi d \eta+\mathcal{O}\left(t^{-5 / 2}\right) \sim_{t \rightarrow 0^{+}} b(y) t^{-3}$, where, $b(y)=\frac{1}{8 \pi} h^{-1 / 2}(0, y) h(0, y)^{3 / 2}\left|\partial_{\theta} y\right|^{2}$.

There are Jacobian factors $\left|\partial_{\theta} y\right|>0$ occuring in (24) because of the change of parametrization of the boundary given by $y \mapsto \theta(y)$ and we also use that $\partial_{\theta} x=0$. Differentiation under the integral sign of the heat parametrix is justified since all exponential sums of derivatives of boundary traces of eigenfunctions are absolutely convergent in view of (20) and derivatives of 
the remainder term are controlled. Just as in (22), the Tauberian theorem gives

$$
c_{22}^{L}(\theta ; \lambda)=\sum_{\lambda_{j} \leq \lambda}\left|\partial_{\theta} v_{j}(\theta)\right|^{2} \sim_{\lambda \rightarrow \infty} c_{22}^{L}(\theta) \lambda^{6}
$$

where,

$$
c_{22}^{L}(\theta)=\frac{1}{48 \pi}[h(0, y(\theta))]\left|\partial_{\theta} y\right|^{2}=(48 \pi)^{-1} .
$$

In (26) we have used that $\theta(y)=\int_{0}^{y}[h(0, s)]^{1 / 2} d s$ (see (15) $)$ and so, $\left|\partial_{\theta} y\right|=$ $[h(0, y(\theta))]^{-1 / 2}$.

Finally, we turn to the mixed sum $c_{12}^{L}(\theta ; \lambda)$. By the same heat analysis as for $c_{22}^{L}(\theta ; \lambda)$, one shows that

$$
\sum_{\lambda_{j} \leq \lambda}\left|\partial_{\theta} v_{j}(\theta)+v_{j}(\theta)\right|^{2} \sim_{\lambda \rightarrow \infty}(48 \pi)^{-1} \lambda^{6}
$$

and so, by writing $2 c_{12}^{L}(\theta ; \lambda)=\sum_{\lambda_{j} \leq \lambda}\left|\left(\partial_{\theta}+1\right) v_{j}(\theta)\right|^{2}-\sum_{\lambda_{j} \leq \lambda}\left|\partial_{\theta} v_{j}(\theta)\right|^{2}-$ $\sum_{\lambda_{j} \leq \lambda}\left|v_{j}(\theta)\right|^{2}$ it follows that $c_{12}^{L}(\theta ; \lambda)=o\left(\lambda^{6}\right)$ uniformly for $\theta \in[0, \ell]$. Unfortunately, this bound is not sufficient to prove our theorem. Indeed, we will need the stronger bound

$$
c_{12}^{L}(\theta ; \lambda)=o\left(\lambda^{5}\right) .
$$

This estimate follows from analysis of the Dirichlet wave kernel and we defer the proof to section 5 (see (49)).

\section{Asymptotics FOR $\mathcal{Z}^{S}(\lambda)$}

5.1. Computation of $c_{11}^{S}(\theta ; \lambda)$. The heat analysis in the previous section is not sufficient to deal with the short-range case, even for the diagonal sums. An alternative approach involves the Dirichlet wave operator. However, unlike the boundaryless case, one does not have an explicit wave parametrix, even for small time. This is the main complication in the case of manifolds with boundary. Indeed, the small-time behaviour of the Dirichlet wave operator kernel $E_{\Omega}(x, y ; t) ; x, y \in \Omega$ is quite complicated and in particular, has no conormal expansion at $t=0$. Nevertheless, it turns out that the restriction to the boundary diagonal does have such an expansion (see (30)). Using the fact that the singularity of $E_{\Omega}^{b}(q, q ; t) ; q \in \partial \Omega$ at $t=0$ is isolated, Zelditch [Z1] computed the asymptotics of $c_{11}^{S}(\theta ; \lambda)$. The idea is to use boundary wave front calculus and the conormal expansion of Ivrii [I] and Melrose [M1 together with the Hadamard variational formulas for the Dirichlet (or Neumann) eigenvalues to compute the coefficients in the conormal expansion of the boundary trace of the interior wave group restricted to the boundary diagonal. The asymptotic expansion of $c_{22}^{S}(\theta ; \lambda)$ and the bound for $c_{12}^{S}(\theta ; \lambda)$ in the next subsection are apparently new. Since the computations for $c_{22}^{S}(\theta ; \lambda)$ and $c_{12}^{S}(\theta ; \lambda)$ are related to the one for $c_{11}^{S}(\theta ; \lambda)$ (and use it), we give the argument for each of spectral sums.

Let

$$
E^{\Omega}(t)=\cos t \sqrt{\Delta_{\Omega}}
$$


be the Dirichlet wave operator where $\Delta_{\Omega}$ denotes the Dirichlet Laplacian on $\Omega$. To fix notation, we denote the boundary restriction operator by $\gamma_{\partial \Omega}$, so that for $u \in \mathcal{D}^{\prime}(\Omega)$ with $W F(u) \cap N^{*} \partial \Omega=\emptyset, \gamma_{\partial \Omega} u$ is the uniquely-defined restriction (ie. pull-back of $u$ ) with $\gamma_{\partial \Omega} u \in \mathcal{D}^{\prime}(\partial \Omega)$ and $\gamma_{\partial \Omega} u=\left.u\right|_{\partial \Omega}$ when $u \in C^{\infty}(\Omega)$.

The diagonal kernel $t^{n} E_{\Omega}(t, x, x) \notin C^{\infty}((0, \epsilon] \times \Omega \times \Omega)$ for any $n>0$ and $\epsilon>0$ when $x \in \Omega$. The singularity at $t=0$ is not even isolated due to reflecting loops based at $x \in \Omega$ with period $t=2 d(x, \partial \Omega)$. However, when $x=q \in \partial \Omega$, the singularity at $t=0$ is isolated. The reason for this can be described as follows: let

$$
\Phi_{t}: T^{*} \Omega \rightarrow T^{*} \Omega
$$

be the time- $t$ broken generalized bicharacteristic flow in $\Omega$ [MSj]. For $(q, \eta) \in$ $B^{*} \partial \Omega$ we let $\xi(q, \eta) \in S_{+}^{*}(\Omega)$ be the unique inward pointing unit vector that projects tangentially onto $\eta$. We denote the tangential projection of $v \in S_{+}^{*}(\Omega)$ here by $v^{T} \in B^{*}(\partial \Omega)$ and $\gamma_{\partial \Omega}:\left.\phi \mapsto \partial_{\nu} \phi\right|_{\partial \Omega}$ the boundary trace operation.

Propagation of singularities in $\Omega$ MSj, M1 gives

$$
W F\left(E_{\Omega}(x, y, t)\right)=\left\{(t, \tau, x, \xi, y, \eta) ; \tau=|\xi|_{g}, \Phi_{t}(x, \xi)=(y, \eta)\right\} .
$$

For fixed $t \in \mathbb{R}$ we define the distribution $E_{\Omega, t}$ on $\Omega \times \Omega$ given by

$$
E_{\Omega, t}(x, y)=E_{\Omega}(x, y, t) \text {. }
$$

Let $N^{*} \Delta_{\partial \Omega}=\{(q, \xi, q,-\xi) ; q \in \partial \Omega\}$ be the conormal space of the diagonal $\Delta_{\partial \Omega} \subset \partial \Omega \times \partial \Omega$. In view of (28), when $t \in(0, \epsilon)$ with $\epsilon>0$ sufficiently small,

$$
W F\left(E_{\Omega, t}\right) \cap N^{*}\left(\Delta_{\partial \Omega}\right)=\emptyset .
$$

This just says that for $|t|$ sufficiently small, there are no non-trivial, periodic broken bicharacteristics passing through $q \in \partial \Omega$ with period $|t|$. Then by wave-front calculus, $E^{b}:=\gamma_{\Delta \partial \Omega} E_{\Omega}$ is well-defined for $\epsilon>0$ small and for $t \in(0, \epsilon)$,

$$
\begin{gathered}
W F\left(E^{b}(q, q, t)\right)=\left\{\left(t, \tau, q, \eta, q, \eta^{\prime}\right) \in T^{*}(-\epsilon, \epsilon) \times B^{*} \partial \Omega \times B^{*} \partial \Omega ; \tau=|\eta|_{g},\right. \\
\left.\left[\Phi_{t}(q, \xi(q, \eta))\right]^{T}=\left(q^{\prime}, \eta^{\prime}\right)\right\} .
\end{gathered}
$$

In (29) $), \xi(q, \eta) \in S_{i n, q}^{*} \Omega$ is the inward-pointwing unit co-vector at $q \in \partial \Omega$ with $[\xi(q, \eta)]^{T}=\eta$ where $v^{T}$ is the tangential projection at $q \in \partial \Omega$.

It follows that the singularity at $t=0$ is classical conormal [I, M1, so that for $\epsilon>0$ small as $t \rightarrow 0^{+}$, one has $t^{n} E^{b}(t, q, q) \in C^{\infty}$ where $n=\operatorname{dim} \Omega$ and so,

$$
t^{n} E^{b}(t, q, q) \sim \sum_{k=0}^{\infty} a_{k}(q) t^{k}
$$

with $a_{j} \in C^{\infty}(\partial \Omega)$. Under the non-recurrence assumption on the billiard flow, the expansion in (30) holds, modulo $C^{\infty}\left(\left(0, \epsilon^{-1}\right) \times \partial \Omega \times \partial \Omega\right)$, for all $t \in\left(0, \epsilon^{-1}\right)$ where $\epsilon>0$ is fixed arbitrarily small.

Since the method of proof in [I, M1 is non-constructive, one needs an additional argument to compute the actual coefficient functions $a_{j}$. One 
way to do this is to integrate both sides in (30) against a test function $\psi \in C^{\infty}(\partial \Omega)$. This is allowed because $t^{n} E^{b} \in C^{\infty}$ and the result is that

$$
t^{n} \sum_{j} e^{i \lambda_{j} t} \int_{\partial \Omega}\left|\partial_{\nu} \phi_{j}(q)\right|^{2} \psi(q) d \sigma(q) \sim_{t \rightarrow 0^{+}} \sum_{k=0}^{\infty} t^{k} \int_{\partial \Omega} a_{k}(q) \psi(q) d \sigma(q) .
$$

Consider a normal variation of the domain $\Omega$ with variation vector field, $\psi \nu$. By taking the variation $\delta_{\psi}$ of both sides in (31) and using the Hadamard variation formula for the eigenvalues:

$$
\delta_{\psi} \lambda_{j}^{2}=2 \lambda_{j} \delta_{\psi} \lambda_{j}=\int_{\partial \Omega} \psi(q)\left|\partial_{\nu} \phi_{j}(q)\right|^{2} d \sigma(q),
$$

it follows that

$$
\begin{gathered}
\frac{1}{i} \frac{\partial}{\partial t}\left(\frac{2}{i t} \sum_{j} \delta_{\psi}\left(e^{i t \lambda_{j}}\right)\right)=\frac{1}{i t} \sum_{j} 2 \lambda_{j} \delta_{\psi}\left(e^{i t \lambda_{j}}\right)=\sum_{j}\left(\delta_{\psi} \lambda_{j}^{2}\right) e^{i \lambda_{j} t} \\
=\sum_{j} e^{i \lambda_{j} t} \int_{\partial \Omega} \psi(q)\left|\partial_{\nu} \phi_{j}(q)\right|^{2} d \sigma(q) .
\end{gathered}
$$

By a well-known asymptotic expansion for the wave trace [I, [M1]

$$
\sum_{j} e^{i \lambda_{j} t} \sim_{t \rightarrow 0^{+}}(2 \pi)^{-2} \operatorname{vol}(\Omega)(t+i 0)^{-2}+C^{\prime} \operatorname{vol}(\partial \Omega)(t+i 0)^{-1}+\ldots
$$

where the dots denote lower-order terms. Substitution of (33) in (32) gives

$$
\frac{1}{i} \frac{\partial}{\partial t}\left(\frac{2}{i t} \sum_{j} \delta_{\psi}\left(e^{i \lambda_{j} t}\right)\right) \sim_{t \rightarrow 0^{+}} 2(2+1)(2 \pi)^{-2} \delta_{\psi} \operatorname{vol}(\Omega)(t+i 0)^{-4}+C^{\prime} \delta_{\psi} \operatorname{vol}(\partial \Omega)(t+i 0)^{-5}+\ldots
$$

Equating coefficiens in (31)-(33) implies that

$$
\sum_{j} e^{i \lambda_{j} t} \int_{\partial \Omega} \psi(q)\left|\partial_{\nu} \phi_{j}(q)\right|^{2} d \sigma(q) \sim_{t \rightarrow 0^{+}} 6(2 \pi)^{-2} \delta_{\psi} \operatorname{vol}(\Omega)(t+i 0)^{-4}+C^{\prime} \delta_{\psi} \operatorname{vol}(\partial \Omega)(t+i 0)^{-3}+\ldots,
$$

where, a direct computation gives

$$
\delta_{\psi} \operatorname{vol}(\Omega)=\int_{\partial \Omega} \psi(q) d \sigma(q) .
$$

Under the assumption that there are measure zero loops at the boundary it follows from (31) by a standard Tauberian argument (see for example [Z1] section 5.2) that

$$
c_{11}^{S}(\theta, \lambda)=\sum_{\lambda_{j} \in[\lambda, \lambda+1]}\left|v_{j}(\theta)\right|^{2}=c_{11}^{S}(\theta) \cdot \lambda^{3}+o\left(\lambda^{2}\right),
$$

and since the test function $\psi \in C^{\infty}(\partial \Omega)$ is arbitrary, it follows from (35) that

$$
c_{11}^{S}(\theta)=(2 \pi)^{-1}
$$


5.2. Computation of $c_{22}^{S}(\theta ; \lambda)$. The computation of $c_{22}^{S}$ uses the asymptotics for $c_{11}^{S}$ above together with some additional integration by parts and applications of the local Weyl law [HZ for boundary traces of eigenfunctions. Let $\partial_{\theta_{q}}: C^{\infty}(\partial \Omega) \rightarrow C^{\infty}(\partial \Omega)$ be the tangential derivative given by $\partial_{\theta_{q}}:=d q\left(\partial_{\theta}\right)$. Since $W F\left(\partial_{\theta_{q}}\right) \subset\left\{(q, \xi ; q,-\xi) ;(q, \xi) \in T^{*} \partial \Omega\right\}$, by wave front calculus,

$$
W F\left(\left[\partial_{\theta_{q}} E_{\Omega} \partial_{\theta_{q}^{\prime}}\right]^{b}\left(q, q^{\prime}, t\right)\right)=W F\left(\left[\partial_{\theta_{q}} E_{\Omega}^{b} \partial_{\theta_{q}^{\prime}}\right]\left(q, q^{\prime}, t\right)\right) \subset W F\left(E_{\Omega}^{b}\left(q, q^{\prime}, t\right)\right) .
$$

Then by the same argument as in the previous section, it follows that $\partial_{\theta_{q}} E_{\Omega}^{b} \partial_{\theta_{q}^{\prime}}$ has a unique restriction to the boundary diagonal which we denote by $\left[\partial_{\theta_{q}} E_{\Omega}^{b} \partial_{\theta_{q}^{\prime}}\right](q, q, t):=\gamma_{\Delta_{\partial \Omega}}\left[\partial_{\theta_{q}} E_{\Omega}^{b} \partial_{\theta_{q}^{\prime}}\right]$. Since wave fronts restrict, under the non-recurrence assumption, for $t \in\left(0, \epsilon^{-1}\right)$ with $\epsilon>0$ arbitrarily small, modulo $C^{\infty}\left(\left(0, \epsilon^{-1}\right) \times \partial \Omega \times \partial \Omega\right)$, one has the following conormal expansion

$$
\left[\partial_{\theta_{q}} E_{\Omega}^{b} \partial_{\theta_{q}^{\prime}}\right](q, q, t) \sim_{t \rightarrow 0^{+}} \sum_{k=0}^{\infty} b_{k}(q) t^{-n-2+k}
$$

where $b_{k} \in C^{\infty}(\partial \Omega)$ and the corresponding two-term asymptotic formula

$$
\sum_{\lambda_{j} \leq \lambda}\left|\partial_{\theta} v_{j}(\theta)\right|^{2}=c_{22}^{S}(\theta) \lambda^{5}+o\left(\lambda^{4}\right)
$$

The remainder of this subsection is devoted to computing the leading coefficient $c_{22}^{S}(\theta)$.

From now on, we put $\hbar_{j}=\frac{1}{\lambda_{j}} ; j=1,2,3, \ldots$ and use semiclassical pseudodifferential calculus on $\partial \Omega$. Let $A_{\hbar_{j}}=O p_{\hbar_{j}}(a)$ with $a \in S_{c l}^{0}\left(T^{*} \partial \Omega\right)$ and $A_{\hbar_{j}}^{q}:=q^{-1} \circ O p_{\hbar_{j}}(a) \circ q$ be the corresponding semiclassical pseudodifferential operator on the parametrizing circle $\mathbb{R} / \ell \mathbb{Z}$. It follows that in the Dirichlet case considered here [HZ],

(40) $\frac{1}{N_{0}(\lambda)} \sum_{\lambda_{j} \leq \lambda}\left\langle\hbar_{j}^{2} A_{\hbar_{j}}^{q} v_{j}, v_{j}\right\rangle=\frac{2}{\pi v o l \Omega} \int_{B^{*} \partial \Omega} a(y, \eta) \sqrt{1-|\eta|_{h}^{2}} d y d \eta+o(1)$,

where, $N_{0}(\lambda):=(2 \pi)^{-2}\left(\right.$ vol $\left.B^{*} \Omega\right) \lambda^{2}$. Since we are assuming here that the measure of periodic broken bicharacteristics is zero, using the conormal expansion in (39) one gets that for some constant $C_{a}$ (yet to be determined),

$$
\frac{1}{N_{0}(\lambda)} \sum_{\lambda_{j} \leq \lambda} \hbar_{j}^{2}\left\langle A_{\hbar_{j}}^{q} v_{j}, v_{j}\right\rangle=C_{a}+o\left(\lambda^{-1}\right)
$$

But then from the weak law in (40) it follows that $C_{a}=\int_{B^{*} \partial \Omega} a(y, \eta) d \sigma(y, \eta)$ where $d \sigma(y, \eta):=2(\pi \operatorname{vol} \Omega)^{-1} \sqrt{1-|\eta|_{h}^{2}} d y d \eta$. Taking sums with $\lambda_{j} \leq \lambda+1$ and $\lambda_{j} \leq \lambda$ in (41) and subtracting them gives

$$
\sum_{\lambda_{j} \in[\lambda, \lambda+1]} \hbar_{j}^{2}\left\langle A_{\hbar_{j}}^{q} v_{j}, v_{j}\right\rangle=\int_{B^{*} \partial \Omega} a(y, \eta) d \sigma(y, \eta)\left[N_{0}(\lambda+1)-N_{0}(\lambda)\right]+o_{A}(\lambda) .
$$


Now rescale and write $\lambda_{j}=\hbar_{j}^{-1}$ and let $\psi \in C^{\infty}(\mathbb{R} / \ell \mathbb{Z})$. In analogy with the previous section, we want to compute

$$
\begin{gathered}
\sum_{\hbar_{j}^{-1} \in[\lambda, \lambda+1]} \int_{0}^{\ell} \psi(\theta)\left|\partial_{\theta} v_{j}(\theta)\right|^{2} d \theta=\sum_{\hbar_{j}^{-1} \in[\lambda, \lambda+1]} \hbar_{j}^{-2}\left\langle\psi\left(\hbar_{j} D_{\theta}\right)^{2} v_{j}, v_{j}\right\rangle \\
+\sum_{\hbar_{j}^{-1} \in[\lambda, \lambda+1]} \hbar_{j}^{-2}\left\langle\left(\hbar_{j} D_{\theta} \psi\right) v_{j}, \hbar_{j} D_{\theta} v_{j}\right\rangle \\
=\sum_{\hbar_{j}^{-1} \in[\lambda, \lambda+1]} \hbar_{j}^{-2}\left\langle\psi\left(\hbar_{j} D_{\theta}\right)^{2} v_{j}, v_{j}\right\rangle+\frac{1}{2} \sum_{\hbar_{j}^{-1} \in[\lambda, \lambda+1]}\left\langle\partial_{\theta}^{2} \psi,\left|v_{j}\right|^{2}\right\rangle \\
=\sum_{\hbar_{j}^{-1} \in[\lambda, \lambda+1]} \hbar_{j}^{-2}\left\langle\psi\left(\hbar_{j} D_{\theta}\right)^{2} v_{j}, v_{j}\right\rangle+\mathcal{O}\left(\lambda^{3}\right) .
\end{gathered}
$$

The second last line in (43) follows by integration by parts and the last line by the result in the previous section for $c_{11}^{S}(\theta ; \lambda)$ (here, we also use that $\left.\hbar_{j}^{-1} \in[\lambda, \lambda+1]\right)$. Since

$$
W F_{\hbar_{j}}^{\prime}\left(F\left(\hbar_{j}^{-1}\right)\right) \subset B_{1+\delta}^{*}(\partial \Omega) \times B_{1+\delta}^{*}(\partial \Omega),
$$

from the boundary jump equation (57) it follows that for any $\delta>0$,

$$
W F_{\hbar_{j}}\left(v_{j}\right) \subset\left(d q^{t}\right)^{-1} B_{1+\delta}^{*}(\partial \Omega) .
$$

Let $\chi \in C_{0}^{\infty}\left(\left(d q^{t}\right)^{-1} B_{1+2 \delta}^{*} \partial \Omega\right)$ with $\chi(\theta, \xi)=1$ for $(\theta, \xi) \in\left(d q^{t}\right)^{-1} B_{1+\delta}^{*} \partial \Omega$. Then, from the last line in (43),

$$
\begin{gathered}
\sum_{\hbar_{j}^{-1} \in[\lambda, \lambda+1]} \hbar_{j}^{-2}\left\langle\psi\left(\hbar_{j} D_{\theta}\right)^{2} v_{j}, v_{j}\right\rangle+\mathcal{O}\left(\lambda^{3}\right) \\
=\sum_{\hbar_{j}^{-1} \in[\lambda, \lambda+1]} \hbar_{j}^{-2}\left\langle O p_{\hbar_{j}}(\chi) \psi\left(\hbar_{j} D_{\theta}\right)^{2} O p_{\hbar_{j}}(\chi) v_{j}, v_{j}\right\rangle+\mathcal{O}\left(\lambda^{3}\right) \\
=\lambda^{4}\left(1+\mathcal{O}\left(\lambda^{-1}\right)\right) \sum_{\hbar_{j}^{-1} \in[\lambda, \lambda+1]} \hbar_{j}^{2}\left\langle O p_{\hbar_{j}}(\chi) \psi\left(\hbar_{j} D_{\theta}\right)^{2} O p_{\hbar_{j}}(\chi) v_{j}, v_{j}\right\rangle+\mathcal{O}\left(\lambda^{3}\right)
\end{gathered}
$$

Clearly, $\psi O p_{\hbar_{j}}(\chi)\left(\hbar_{j} D_{\theta}\right)^{2} O p_{\hbar_{j}}(\chi) \in O p_{\hbar_{j}}\left(S_{c l}^{0}\right)$ has principal symbol

$$
\sigma\left[\psi O p_{\hbar_{j}}(\chi)\left|\hbar_{j} D_{\theta}\right|^{2} O p_{\hbar_{j}}(\chi)\right](\theta, \xi)=\psi(\theta)|\xi|^{2},
$$

for $(\theta, \xi) \in\left(d q^{t}\right)^{-1} B^{*} \partial \Omega$. But then, since $\hbar_{j}^{-1} \in[\lambda, \lambda+1]$, it follows from (42) that one has the two-term asymptotic formula $c_{22}^{S}(\theta ; \lambda)=c_{22}^{S}(\theta) \lambda^{5}+o\left(\lambda^{4}\right)$, where,

$$
c_{22}^{S}(\theta)=\lim _{\lambda \rightarrow \infty} \lambda^{-5} \frac{2}{\pi \operatorname{vol} \Omega}\left(\int_{|\xi| \leq 1}|\xi|^{2} \sqrt{1-|\xi|^{2}} d \xi\right) \lambda^{4}\left[N_{0}(\lambda+1)-N_{0}(\lambda)\right],
$$

and since $N_{0}(\lambda+1)-N_{0}(\lambda)=2(2 \pi)^{-2}\left(\operatorname{vol} B^{*} \Omega\right) \lambda$, it follows that

$$
c_{22}^{S}(\theta)=2 \times \frac{\pi}{8} \times \frac{2}{\pi \operatorname{vol} \Omega} \times(2 \pi)^{-2} \operatorname{vol} B^{*} \Omega=(8 \pi)^{-1} .
$$


5.3. Bound for $c_{12}^{S}(\theta ; \lambda)$. By the same argument as for $c_{22}^{S}(\theta ; \lambda)$ one gets the two-term asymptotic formula,

$$
\sum_{\lambda_{j} \in[\lambda, \lambda+1]}\left|\partial_{\theta} v_{j}(\theta)+v_{j}(\theta)\right|^{2}=(8 \pi)^{-1} \lambda^{5}+o\left(\lambda^{4}\right),
$$

uniformly for $\theta \in[0, \ell]$. Then,

$$
2 c_{12}^{S}(\theta ; \lambda)=\sum_{\lambda_{j} \in[\lambda, \lambda+1]}\left|\partial_{\theta} v_{j}(\theta)+v_{j}(\theta)\right|^{2}-\sum_{\lambda_{j} \in[\lambda, \lambda+1]}\left|\partial_{\theta} v_{j}(\theta)\right|^{2}-\sum_{\lambda_{j} \in[\lambda, \lambda+1]}\left|v_{j}(\theta)\right|^{2}=o\left(\lambda^{4}\right),
$$

where, the final bound in (47) follows from the two-term asymptotic formulas in (37), (45) and (47). The upshot is that

$$
c_{12}^{S}(\theta ; \lambda)=o\left(\lambda^{4}\right)
$$

and so, by the triangle inequality,

$$
\left|c_{12}^{L}(\theta ; \lambda)\right| \leq C_{\Omega} \lambda\left|c_{12}^{S}(\theta ; \lambda)\right|=o\left(\lambda^{5}\right) .
$$

\section{Concluding the Proof of theorem 1.1}

Corollary 6.1. There exists a constant $\lambda_{0}>0$ such that for $\lambda \geq \lambda_{0}$ the vectors $b_{1}^{L, S}(\theta ; \lambda)$ and $b_{2}^{L, S}(\theta ; \lambda)$ are linearly independent for all $\theta \in[0, \ell]$.

Proof. By Cauchy-Schwarz, the vectors $b_{1}^{L, S}(\theta ; \lambda)=\left(v_{1}(\theta), \ldots ., v_{\lambda}(\theta)\right)$ and $b_{1}^{L, S}(\theta ; \lambda)=\left(\partial_{\theta} v_{1}(\theta), \ldots ., \partial_{\theta} v_{\lambda}(\theta)\right)$ are collinear for some $\theta \in[0, \ell]$ if and only if

$$
c_{11}^{L, S}(\theta ; \lambda) \cdot c_{22}^{L, S}(\theta ; \lambda)-\left|c_{12}^{L, S}(\theta ; \lambda)\right|^{2}=0 .
$$

But by our pointwise Weyl sum computations in (23), (26) and (27),

$$
c_{11}^{L}(\theta ; \lambda) \cdot c_{22}^{L}(\theta ; \lambda)-\left|c_{12}^{L}(\theta ; \lambda)\right|^{2} \sim_{\lambda \rightarrow \infty} C_{\Omega}(\theta) \lambda^{10} ; C_{\Omega}(\theta)>0,
$$

and similarily, from (36), (44) and (48),

$$
c_{11}^{S}(\theta ; \lambda) \cdot c_{22}^{S}(\theta ; \lambda)-\left|c_{12}^{S}(\theta ; \lambda)\right|^{2} \sim_{\lambda \rightarrow \infty} \tilde{C}_{\Omega}(\theta) \lambda^{8} ; \tilde{C}_{\Omega}(\theta)>0 .
$$

So, for $\lambda>0$ large, neither expression vanishes.

Remark 6.2 . We note that by a similar analysis to the one given in sections 4 and 5 and Corollary 6.1 above, one easily shows that the vectors $\left(\partial_{\theta} v_{j}(\theta)\right)_{\lambda_{j} \in[\lambda, \lambda+1]}$ and $\left(\partial_{\theta}^{2} v_{j}(\theta)\right)_{\lambda_{j} \in[\lambda, \lambda+1]}$ are linearly independent for each $\theta \in[0, \ell]$. The analogous result for the long-range case is also valid.

Proof of theorem 1.1. Combining the asymptotic formulas for $c_{i j}^{L, S}(\theta ; \lambda), i, j=$ 1,2 in (23), (26) and (27) and (37), (45) and (48) gives

$$
\left(\frac{c_{12}^{L, S}(\theta ; \lambda)}{c_{11}^{L, S}(\theta ; \lambda)}\right)^{2}=o(\lambda)
$$

uniformly for $\theta \in[0, \ell]$. On the other hand,

$$
\frac{c_{22}^{L, S}(\theta ; \lambda)}{c_{11}^{L, S}(\theta ; \lambda)} \sim_{\lambda \rightarrow \infty} \frac{c_{22}^{L, S}(\theta)}{c_{11}^{L, S}(\theta)} \lambda^{2}
$$

where,

$$
\frac{c_{22}^{L}(\theta)}{c_{11}^{L}(\theta)}=\frac{1}{6} \text { and } \quad \frac{c_{22}^{S}(\theta)}{c_{11}^{S}(\theta)}=\frac{1}{4}
$$


So, substitution into the Kac-Rice formula in Proposition 3.2

$$
\mathcal{Z}^{L, S}(\lambda)=\frac{1}{\pi}\left(\int_{0}^{\ell}\left|\frac{c_{22}^{L, S}(\theta)}{c_{11}^{L, S}(\theta)}\right|^{\frac{1}{2}} d \theta\right) \lambda+o(\lambda),
$$

implies that $\mathcal{Z}^{S}(\lambda)=\frac{\ell(\partial \Omega)}{2 \pi} \lambda+o(\lambda)$ and $\mathcal{Z}^{L}(\lambda)=\frac{\ell(\partial \Omega)}{\sqrt{6} \pi} \lambda+o(\lambda)$.

\section{Appendix A. Proof of the KaC-Rice formula}

Proof of Lemma 3.1. The proof in the long and short range cases is the same, so without loss of generality we treat the long range case here.

In the following, we fix $\lambda>0$ and put $N=N^{L}(\lambda)$. Consider the map

$$
\begin{gathered}
\Psi_{\lambda}: \mathbb{R}^{N} \times[0, \ell] \rightarrow \mathbb{R}^{2} \\
(a, \theta) \mapsto\left(V_{a}^{L}(\theta ; \lambda), \partial_{\theta} V_{a}^{L}(\theta ; \lambda)\right) .
\end{gathered}
$$

Clearly,

$$
\mathcal{C}=\pi_{1}\left(\Psi_{\lambda}^{-1}(0,0)\right)
$$

where $\pi_{1}$ is the projection onto $\mathbb{R}^{N}$. We claim that $\Psi_{\lambda}$ is a submersion. Given this, one has

$$
\operatorname{dim} \Psi_{\lambda}^{-1}(0,0)=N-1,
$$

and so, $\operatorname{dim} \mathcal{C} \leq N-1$, which proves the Lemma.

To see that $\Psi_{\lambda}$ is a submersion, we note that its $(N+1) \times 2$ differential matrix is given by

$$
d \Psi_{\lambda}(a, \theta)=\left(\begin{array}{cc}
b_{1}^{L}(\theta ; \lambda) & b_{2}^{L}(\theta ; \lambda) \\
* & *
\end{array}\right)
$$

where $b_{1}^{L}(\theta ; \lambda)$ and $b_{2}^{L}(\theta ; \lambda)$ are the vectors introduced in section 3.1. The matrix $d \Psi_{\lambda}$ is of full rank (i.e. rank 2 ) for $\lambda$ sufficiently large, by Corollary 6.1 and so $\Psi_{\lambda}$ is a submersion.

Definition 1. Let $\chi_{[-1,1]}$ be the characteristic function of the interval $[-1,1]$ and fix $\epsilon>0$. We introduce the random variables

$$
\tilde{\mathcal{I}}_{a, \epsilon}^{L, S}(\lambda):=\frac{1}{2 \epsilon} \int_{0}^{\ell} \chi_{[-1,1]}\left(\frac{V_{a}^{L, S}(\theta ; \lambda)}{\epsilon}\right)\left|\partial_{\theta} V_{a}^{L, S}(\theta ; \lambda)\right| d \theta .
$$

When $V_{a}^{L, S}(\cdot ; \lambda)$ has no double zeros,

$$
\lim _{\epsilon \rightarrow 0^{+}} \tilde{\mathcal{I}}_{a, \epsilon}^{L, S}(\lambda)=\tilde{\mathcal{I}}_{a}^{L, S}(\lambda) .
$$

To compute $\mathbb{E} \tilde{\mathcal{I}}_{a}^{L, S}(\lambda)$, we need to interchange the $\epsilon \rightarrow 0^{+}$limit and integration over $a \in \mathbb{R}^{N^{L, S}(\lambda)}$. This requires showing that $\tilde{\mathcal{I}}_{a, \epsilon}^{L, S}(\lambda)$ is bounded uniformly in $\epsilon>0$ by a $\mu$-integrable function in the $a$-variables. 
Lemma A.1. Fix $\lambda>0$ sufficiently large and let $\tilde{\mathcal{I}}_{a, \epsilon}^{L, S}(\lambda)$ be defined as in (501).

(i) In the case where $\partial \Omega$ is $C^{\infty}$,

$$
\sup _{\epsilon \in\left[0, \epsilon_{0}\right]} \tilde{\mathcal{I}}_{a, \epsilon}^{L, S}(\lambda) \in L^{1}\left(\mathbb{R}^{N^{L, S}(\lambda)} ; e^{-\|a\|^{2} / 2} \frac{d a}{(2 \pi)^{N^{L, S}(\lambda) / 2}}\right) .
$$

(ii) In the case where $\partial \Omega$ is $C^{\omega}$, one has the explicit bound

$$
\sup _{\epsilon \in\left[0, \epsilon_{0}\right]} \tilde{\mathcal{I}}_{a, \epsilon}^{L, S}(\lambda) \leq C_{\Omega}^{L, S} \lambda,
$$

where, $C_{\Omega}^{L, S}>0$ are constants depending only on $\Omega$.

Proof. (i) The argument for both the long and short ranges are the same, so without loss of generality, we assume here that $\lambda_{j} \in[\lambda, \lambda+1]$. To prove the first part of the Lemma, only the behaviour in $a \in \mathbb{R}^{N^{S}}$ is important (and not in $\lambda$ ). So, we henceforth fix $\lambda>0$ sufficiently large and suppress dependence of all sets, functions, etc. on $\lambda$. In the smooth case, the asymptotics in $\lambda$ of the number of boundary critical points is, to our knowledge, open and probably rather difficult (see however part (ii) and [TZ1] for a sharp result in $\lambda$ in the real-analytic case).

First, we note that since $V_{a}^{S}(\theta ; \lambda)=\sum_{\lambda_{j} \in[\lambda, \lambda+1]} a_{j} v_{j}(\theta)$ is linear in $a \in$ $\mathbb{R}^{N^{S}}$, the number of boundary critical points, $C\left(V_{a} ; \lambda\right)$, is invariant under scaling by $\|a\| \neq 0$; that is,

$$
\mathcal{C}\left(V_{a} ; \lambda\right)=\mathcal{C}\left(V_{\omega} ; \lambda\right)
$$

where, $\omega:=\frac{a}{\|a\|}$. Thus, we are reduced to proving that

$$
\mathcal{C}\left(V_{\omega} ; \lambda\right) \in L^{1}\left(\mathbb{S}^{N^{S}-1} ; d \omega\right),
$$

where $d \omega$ is the standard, unit constant curvature hypersurface measure on $\mathbb{S}^{N^{S}-1}$.

The second point is that by the same argument as in the previous Lemma 3.1 (see also Corollary 6.1 and Remark 6.2), with $V_{a}$ (resp. $\partial_{\theta} V_{a}$ ) replaced by $\partial_{\theta} V_{\omega}$ (resp. $\partial_{\theta}^{2} V_{\omega}$ ) one shows that given the map $d_{\theta} \Psi_{\lambda}: \mathbb{S}^{N^{S}-1} \times[0, \ell] \rightarrow \mathbb{R}^{2}$ defined by

$$
d_{\theta} \Psi_{\lambda}:(\omega, \theta) \mapsto\left(\partial_{\theta} V_{\omega}(\theta ; \lambda), \partial_{\theta}^{2} V_{\omega}(\theta ; \lambda)\right),
$$

the set $d_{\theta} \Psi_{\lambda}^{-1}(0,0) \subseteq \mathbb{S}^{N^{S}-1} \times \mathbb{R}$ is a finite union of compact, $C^{\infty}$ hypersurfaces (here, we use that $V_{\omega}(\theta+\ell ; \lambda)=V_{\omega}(\theta ; \lambda)$ for all $\left.\theta \in \mathbb{R}\right)$. But then, since $\mathbb{S}^{N^{S}-1} \times \mathbb{R} \cong \mathbb{R}^{N^{S}}-0$, by the generalized Jordan-Brouwer separation theorem applied to each of the compact, connected hypersurfaces [A], it follows that $\left(\mathbb{S}^{N^{S}-1} \times \mathbb{R}\right)-d_{\theta} \Psi_{\lambda}^{-1}(0,0)$ has finitely-many connected components. Now consider

$$
\mathcal{C}^{\prime}:=\left\{\omega \in \mathbb{S}^{N^{S}-1} ; \exists \theta \in[0, \ell], \partial_{\theta} V_{\omega}(\theta ; \lambda)=\partial_{\theta}^{2} V_{\omega}(\theta ; \lambda)=0\right\} .
$$

Written another way, $\mathcal{C}^{\prime}=\pi\left(d_{\theta} \Psi_{\lambda}^{-1}(0,0)\right)$ where $\pi: \mathbb{S}^{N^{S}-1} \times \mathbb{R} \rightarrow \mathbb{S}^{N^{S}-1}$ is the smooth canonical projection map $\pi:(\omega, \theta) \mapsto \omega$. Since $\pi$ maps connected sets to connected sets, it follows that

$$
H^{0}\left(\mathbb{S}^{N^{S}-1}-\mathcal{C}^{\prime}\right)<\infty
$$


and clearly, $\omega\left(\mathcal{C}^{\prime}\right)=0$. Now, make the decomposition $\mathbb{S}^{N^{S}-1}-\mathcal{C}^{\prime}=B_{1} \cup \cdots \cup$ $B_{M} ; M<\infty$ where the $B_{j}$ 's are the (open) connected components (which, implicitly depend on $\lambda$.) Without any loss in generality, we choose a point $\omega_{0} \in B_{1}$. Then, all boundary critical points of $V_{\omega_{0}}(\theta ; \lambda)$ are simple and we denote them by $\theta_{0}, \ldots, \theta_{P}$ where $P$ is finite (again, implicitly dependent on $\lambda$ ). Since the following argument is the same for each of the critical points, we consider the first one: $\theta=\theta_{0}$. For $\left(\theta_{0}, \omega_{0}\right) \in[0, \ell] \times \mathbb{S}^{N^{S}-1}$, we have by definition

$$
\partial_{\theta} V_{\omega_{0}}\left(\theta_{0} ; \lambda\right)=0,
$$

and since $\omega_{0} \in B_{1} \subset \mathbb{S}^{N^{S}-1}-\mathcal{C}^{\prime}$, it follows that

$$
\partial_{\theta}^{2} V_{\omega_{0}}\left(\theta_{0} ; \lambda\right) \neq 0 \text {. }
$$

Then, by the implicit function theorem, it follows that for $\omega \in B_{1}$, there is a unique $C^{\infty}$ family of solutions

$$
\left(\omega, \theta_{0}(\omega)\right) \in B_{1} \times[0, \ell]
$$

to (53) satisfying $\theta_{0}\left(\omega_{0}\right)=\theta_{0}$. Repeating the same implicit function theorem argument for the other zeros gives the existence of smooth families of solutions $\theta_{1}(\omega), \ldots, \theta_{P}(\omega)$ to (53) for $\omega \in B_{1}$ with respective initial conditions $\theta_{1}, \ldots, \theta_{P}$. One can apply the same analysis to each of the other connected components $B_{2}, \ldots, B_{M}$. Let

$$
B_{k, m}=\left\{\omega \in B_{k}: \mathcal{C}\left(V_{\omega} ; \lambda\right)=m\right\},
$$

so that

$$
B_{k}=\bigcup_{m=0}^{\infty} B_{k, m} .
$$

The argument above implies that each of the $B_{k, m}$ is open, and thus also closed (being the complement of $\bigcup_{m^{\prime} \neq m} B_{k, m^{\prime}}$ ) in $B_{k}$. This implies that for each $m$, either $B_{k, m}=B_{k}$ or $B_{k, m}=\emptyset$. We conclude that $\mathcal{C}\left(V_{\omega} ; \lambda\right)$ is constant (and finite) on each of the (finitely-many) connected components $B_{k}$ of the $N-1$ sphere. It follows that $\mathcal{C}\left(V_{\omega} ; \lambda\right) \in L^{1}\left(\mathbb{S}^{N^{S}-1} ; d \omega\right)$ and so also,

$$
\mathcal{C}\left(V_{a} ; \lambda\right) \in L^{1}\left(\mathbb{R}^{N^{L, S}(\lambda)} ; e^{-\|a\|^{2} / 2} \frac{d a}{(2 \pi)^{N^{L, S}(\lambda) / 2}}\right) .
$$

So, given (55), it suffices to prove that $\tilde{\mathcal{I}}_{a, \epsilon}^{S}(\lambda)=\mathcal{O}\left(\mathcal{C}\left(V_{a} ; \lambda\right)\right)$ uniformly in $\epsilon>0$. The domain of integration in the definition (50) of $\tilde{\mathcal{I}}_{\epsilon}^{S}(\lambda)$ is a finite union $[0, \ell]=\bigcup_{j=1}^{n} I_{j}$ of disjoint intervals $I_{j}=\left[\left(c_{j}, d_{j}\right)\right]$ each containing at most one critical point. It is clear that the contribution of each of the $I_{j}$ to the integral (50) is at most 2. Moreover, for each $j$ one of the following holds:

(1) $c_{j}=0$.

(2) $d_{j}=\ell$

(3) $\partial_{\theta} V_{a}^{S}(\theta ; \lambda)=0$ for some $\theta \in I_{j}$.

(4) Either $\left(V_{a}^{S}\left(c_{j} ; \lambda\right)=-\epsilon\right.$ and $\left.V_{a}^{S}\left(d_{j} ; \lambda\right)=\epsilon\right)$ or $\left(V_{a}^{S}\left(c_{j}, \lambda\right)=\epsilon\right.$ and $\left.V_{a}^{S}\left(d_{j} ; \lambda\right)=-\epsilon\right)$. 
In the latter case, if $j<n$, then $V_{a}^{S^{\prime}}(\theta ; \lambda)=0$ for some $\theta \in\left[d_{j}, c_{j+1}\right]$. Therefore, $n \leq 2+\mathcal{C}\left(V_{a} ; \lambda\right)+\left(\mathcal{C}\left(V_{a} ; \lambda\right)+1\right)=2 \mathcal{C}\left(V_{a} ; \lambda\right)+3$. Thus the integral in (50) is bounded by

$$
\tilde{\mathcal{I}}_{a, \epsilon}^{S}(\lambda) \leq 2 \cdot n \leq 2 \cdot\left(2 \mathcal{C}\left(V_{a} ; \lambda\right)+3\right)=\mathcal{O}\left(\mathcal{C}\left(V_{a} ; \lambda\right)\right) .
$$

(ii) Although we will not need the much stronger analytic bound in Lemma A.1 (ii) in the current paper, we give the proof here since in light of recent work of Toth and Zelditch [TZ1, we think it is of independent interest. From [TZ1] Theorems 1-3, one has the following bound for the number of boundary critical points of individual eigenfunctions, $v_{j}$ :

$$
\mathcal{C}\left(v_{j}\right) \leq C_{\Omega} \lambda_{j} .
$$

Let $F(\lambda): C^{\infty}(\partial \Omega) \rightarrow C^{\infty}(\partial \Omega)$ be defined by

$$
F(\lambda) f(q)=\int_{\partial \Omega} \partial_{\nu_{q}} G_{0}\left(q, q^{\prime} ; \lambda\right) f\left(q^{\prime}\right) d \sigma\left(q^{\prime}\right)
$$

where $G_{0}\left(q, q^{\prime} ; \lambda\right)=\frac{i}{4} \mathrm{Ha}_{0}^{(1)}\left(\lambda\left|q-q^{\prime}\right|\right)$ is the free outgoing Greens function for the Helmholtz equation in $\mathbb{R}^{2}$. Roughly speaking, the bound in (56) follows by holomorphically continuing both sides of the jumps-equation

$$
\left.\partial_{\nu} \phi_{j}\right|_{\partial \Omega}=-2 F\left(\lambda_{j}\right)\left(\left.\partial_{\nu} \phi_{j}\right|_{\partial \Omega}\right)
$$

and using a Jensen-type argument to bound the number of complex (and hence real) zeros in a complex tube $\partial \Omega_{\mathbb{C}}$ containing $\partial \Omega$ by the exponential growth exponent of the holomorphically continued $F(\lambda)$-kernel. Let $v_{j}^{\mathbb{C}}$ denote the holomorphic continuation of $v_{j}$ to complex parameter strip $A(\epsilon)$ : $[0, \ell] \times[-\epsilon, \epsilon]$ where the holomorphic continuation of $q$ is $q^{\mathbb{C}}: A(\epsilon) \rightarrow \partial \Omega_{\mathbb{C}}$. Then, one has the exponential growth estimate [TZ1]

$$
\sup _{\zeta \in A(\epsilon)}\left|v_{j}^{\mathbb{C}}(\zeta)\right| \leq \exp \left[C_{\Omega}|\Im \zeta| \lambda_{j}\right]
$$

Moreover,

$$
V_{a}^{\mathbb{C}}(\zeta)=\sum_{\lambda_{j} \in[\lambda, \lambda+1]} a_{j} v_{j}^{\mathbb{C}}(\zeta)
$$

and so by (58) and Cauchy-Schwartz,

$$
\left|V_{a}^{\mathbb{C}}(\zeta)\right| \leq \sqrt{N^{S}(\lambda)}\|a\| \exp \left[C_{\Omega}|\Im \zeta| \lambda\right]
$$

Since $N^{S}(\lambda) \sim \lambda$, Theorem 3 in [TZ1] applied to $\Phi_{a}^{\mathbb{C}}$ gives

$$
\mathcal{C}\left(V_{a} ; \lambda\right) \leq C_{\Omega} \max _{\zeta \in \partial \Omega_{\mathbb{C}}}|\log | V_{a}^{\mathbb{C}}(\zeta)|| \leq C_{\Omega}^{\prime}(\lambda+|\log \|a\||)
$$

in view of (59). But by the scaling invariance (51), it suffices to assume that $\|a\|=1$, so the last bound in (60) is $\mathcal{O}_{\Omega}(\lambda)$. Now, just as in (i) one uses that $\sup _{\epsilon} \tilde{\mathcal{I}}_{a, \epsilon}^{S}(\lambda)=\mathcal{O}\left(\mathcal{C}\left(V_{a} ; \lambda\right)\right)$. Again, the same argument works in the long-range case.

Proof of Proposition 3.2. We use (8) so that we are to compute the expected number $\mathbb{E} \tilde{\mathcal{I}}^{L, S}(\lambda)$ of the zeros of $V_{a}^{L, S}(\cdot ; \lambda)$, defined by (6) and (7) on $[0, \ell]$. 
By Lemma 3.1 we can assume that $V_{a}^{L, S}(\theta ; \lambda)$ has no double zeros so that

$$
\tilde{\mathcal{I}}_{a}^{L, S}(\lambda)=\lim _{\epsilon \rightarrow 0} \frac{1}{2 \epsilon} \int_{0}^{\ell} \chi_{[-1,1]}\left(\frac{V_{a}^{L, S}(\theta ; \lambda)}{\epsilon}\right)\left|\partial_{\theta} V_{a}^{L, S}(\theta ; \lambda)\right| d \theta,
$$

and so,

$$
\mathbb{E} \tilde{\mathcal{I}}_{a}^{L, S}(\lambda)=\mathbb{E} \lim _{\epsilon \rightarrow 0} \frac{1}{2 \epsilon} \int_{0}^{\ell} \chi_{[-1,1]}\left(\frac{V_{a, \epsilon}^{L, S}(\theta ; \lambda)}{\epsilon}\right)\left|\partial_{\theta} V_{a}^{L, S}(\theta ; \lambda)\right| d \theta .
$$

Since by Lemma A.1, for each fixed $\lambda \in \mathbb{R}^{+}$sufficiently large, the function

$$
\sup _{\epsilon} \tilde{\mathcal{I}}_{a, \epsilon}^{L, S}(\lambda) \in L^{1}\left(\mathbb{R}^{N^{L, S}(\lambda)} ; e^{-\|a\|^{2} / 2} \frac{d a}{(2 \pi)^{N^{L, S}(\lambda) / 2}}\right),
$$

by dominated convergence, we interchange the order of the integration and limit and get

$$
\begin{aligned}
\mathbb{E} \tilde{\mathcal{I}}_{a}^{L, S}(\lambda) & =\lim _{\epsilon \rightarrow 0} \frac{1}{2 \epsilon} \mathbb{E} \int_{0}^{\ell} \chi_{[-1,1]}\left(\frac{V_{a}^{L, S}(\theta ; \lambda)}{\epsilon}\right)\left|\partial_{\theta} V_{a}^{L, S}(\theta ; \lambda)\right| d \theta \\
& =\lim _{\epsilon \rightarrow 0} \int_{0}^{\ell} \mathbb{E}\left[\frac{1}{2 \epsilon} \chi_{[-1,1]}\left(\frac{V_{a}^{L, S}(\theta ; \lambda)}{\epsilon}\right)\left|\partial_{\theta} V_{a}^{L, S}(\theta ; \lambda)\right|\right] d \theta,
\end{aligned}
$$

by Fubini.

We rewrite the last equality as

$$
\mathbb{E} \tilde{\mathcal{I}}_{a}^{L, S}(\lambda)=\lim _{\epsilon \rightarrow 0} \int_{0}^{\ell} K_{\epsilon}^{L, S}(\theta ; \lambda) d \theta
$$

where

$$
K_{\epsilon}^{L, S}(\theta ; \lambda)=\mathbb{E}\left[\frac{1}{2 \epsilon} \chi_{[-1,1]}\left(\frac{V_{a}^{L, S}(\theta ; \lambda)}{\epsilon}\right)\left|\partial_{\theta} V_{a}^{L, S}(\theta ; \lambda)\right|\right] .
$$

Assuming $\lambda$ is fixed, we denote $N=N^{L, S}(\lambda)$. To compute $K_{\epsilon}^{L, S}(\theta ; \lambda)$ for a given $\theta \in[0, \ell]$, we note that

$$
\left\langle b_{1}^{L, S}(\theta ; \lambda), a\right\rangle=V_{a}^{L, S}(\theta ; \lambda)
$$

and

$$
\left\langle b_{2}^{L, S}(\theta ; \lambda), a\right\rangle=\partial_{\theta} V_{a}^{L, S}(\theta ; \lambda) .
$$

By Corollary 6.1 for $\lambda$ large, $b_{1}^{L, S}(\theta ; \lambda)$ and $b_{2}^{L, S}(\theta ; \lambda)$ are nowhere collinear and so the vectors $\left\{b_{1}^{L, S}(\theta ; \lambda), b_{2}^{L, S}(\theta ; \lambda)\right\}$ can be extended to a basis

$$
\left\{b_{1}^{L, S}(\theta ; \lambda), b_{2}^{L, S}(\theta ; \lambda), b_{3}^{L, S}(\theta ; \lambda), \ldots, b_{N}^{L, S}(\theta ; \lambda)\right\}
$$

of $\mathbb{R}^{N}$ with the property that $\left\{b_{3}^{L, S}(\theta ; \lambda), \ldots, b_{N}^{L, S}(\theta ; \lambda)\right\}$ is an orthonormal basis of $\operatorname{span}\left\{b_{1}^{L, S}(\theta ; \lambda), b_{2}^{L, S}(\theta ; \lambda)\right\}^{\perp}$. Let $B^{L, S}(\theta ; \lambda) \in M_{N}(\mathbb{R})$ be the matrix with row vectors $b_{k}^{L, S}(\theta ; \lambda)$. Then

$$
B^{L, S}(\theta ; \lambda) B^{L, S}(\theta ; \lambda)^{t}=\left(\begin{array}{cc}
C^{L, S}(\theta ; \lambda) & 0 \\
0 & I_{N-2}
\end{array}\right)
$$


where

$$
C^{L, S}(\theta ; \lambda)=\left(\begin{array}{cc}
c_{11}^{L, S}(\theta ; \lambda) & c_{12}^{L, S}(\theta ; \lambda) \\
c_{21}^{L, S}(\theta ; \lambda) & c_{22}^{L, S}(\theta ; \lambda)
\end{array}\right)
$$

with $c_{i j}$ being defined in section 3.1. In particular,

$$
\operatorname{det} B^{L, S}(\theta ; \lambda)=\sqrt{\operatorname{det} C^{L, S}(\theta ; \lambda)} .
$$

Writing the Gaussian probability density explicitly in (62) yields the formula

$$
K_{\epsilon}^{L, S}(\theta ; \lambda)=\frac{1}{2 \epsilon} \int \chi_{[-1,1]}\left(\frac{\left\langle b_{1}^{L, S}(\theta ; \lambda), a\right\rangle}{\epsilon}\right)\left|\left\langle b_{2}^{L, S}(\theta ; \lambda), a\right\rangle\right| \exp \left(-\frac{1}{2}\|a\|^{2}\right) \frac{d a_{1} \cdots d a_{N}}{(2 \pi)^{N / 2}}
$$

We change the variables $v=a B$. In the new coordinates, we have

$$
\begin{aligned}
\|a\|^{2} & =a \cdot a^{t}=v B^{L, S}(\theta ; \lambda)^{-1}\left(B^{L, S}(\theta ; \lambda)^{-1}\right)^{t} v^{t} \\
& =v\left(\begin{array}{cc}
C^{L, S}(\theta ; \lambda) & 0 \\
0 & I_{N-2}
\end{array}\right)^{-1} v^{t}=w_{1} C^{L, S}(\theta ; \lambda)^{-1} w_{1}^{t}+\left\|w_{2}\right\|^{2},
\end{aligned}
$$

where $w_{1}=\left(v_{1}, v_{2}\right)$ and $w_{2}=\left(v_{3}, \ldots, v_{N}\right)$, so that

$$
\begin{aligned}
K_{\epsilon}^{L, S}(\theta ; \lambda) & =\frac{1}{(2 \pi)^{N / 2} \sqrt{\operatorname{det} C^{L, S}(\theta ; \lambda)}} \times \\
& \times \int_{\mathbb{R}^{N}} \frac{1}{2 \epsilon} \chi_{[-1,1]}\left(\frac{v_{1}}{\epsilon}\right)\left|v_{2}\right| \exp \left(-\frac{1}{2}\left(w_{1} C^{L, S}(\theta ; \lambda)^{-1} w_{1}^{t}+\left\|w_{2}\right\|^{2}\right)\right) d v_{1} \cdots d v_{N} \\
& =\frac{1}{(2 \pi) \sqrt{\operatorname{det} C^{L, S}(\theta ; \lambda)}} \int_{\mathbb{R}^{2}} \frac{1}{2 \epsilon} \chi_{[-1,1]}\left(\frac{v_{1}}{\epsilon}\right)\left|v_{2}\right| \exp \left(-\frac{1}{2} w_{1} C^{L, S}(\theta ; \lambda)^{-1} w_{1}^{t}\right) d w_{1} \times \\
& \times \int_{\mathbb{R}^{N-2}} \exp \left(-\frac{1}{2}\left\|w_{2}\right\|^{2}\right) \frac{d w_{2}}{(2 \pi)^{\frac{N-2}{2}}} .
\end{aligned}
$$

Note that the last integrand is just the standard multivariate Gaussian probability measure, so that the corresponding integral is just 1 .

Therefore, we get the formula

$$
\begin{aligned}
K_{\epsilon}^{L, S}(\theta ; \lambda) & =\frac{1}{2 \pi \sqrt{\operatorname{det} C^{L, S}(\theta ; \lambda)}} \times \\
& \times \frac{1}{2 \epsilon} \int_{-\infty}^{\infty} \int_{-\epsilon}^{\epsilon}\left|v_{2}\right| \exp \left(-\frac{1}{2}\left(v_{1}, v_{2}\right) C^{L, S}(\theta ; \lambda)^{-1}\left(v_{1}, v_{2}\right)^{t}\right) d v_{1} d v_{2} .
\end{aligned}
$$

We wish to apply the dominated convergence theorem again to exchange the limit and the integral in (61). For this we estimate $K_{\epsilon}^{L, S}(\theta ; \lambda)$ from above in the following way. Let $E=\left\{e_{i}\right\}$ be any orthonormal basis of $\mathbb{R}^{N}$, with $e_{1}=\frac{b_{1}}{\left\|b_{1}\right\|}$. Then for $a=E a^{\prime}$ the integral in (64) is, using the invariance of the Gaussian measure,

$$
\frac{1}{2 \epsilon} \int_{\mathbb{R}^{N}} \chi_{[-1,1]}\left(\frac{\left\langle b_{1}^{L, S}(\theta, \lambda), a_{1}^{\prime} e_{1}\right\rangle}{\epsilon}\right)\left|\left\langle b_{2}^{L, S}(\theta, \lambda), E a^{\prime}\right\rangle\right| \exp \left(-\frac{1}{2}\left\|a^{\prime}\right\|^{2}\right) \frac{d a^{\prime}}{(2 \pi)^{N / 2}} .
$$


We have

$$
\left\langle b_{1}^{L, S}(\theta ; \lambda), a_{1}^{\prime} e_{1}\right\rangle=a_{1}^{\prime}\left\|b_{1}^{L, S}(\theta ; \lambda)\right\|=a_{1}^{\prime} \sqrt{c_{11}},
$$

so that we integrate for $\left|a_{1}^{\prime}\right| \leq \frac{\epsilon}{\sqrt{c_{11}^{L, S}(\theta ; \lambda)}}$, and we have by the Cauchy-

Schwartz inequality

$$
\left|\left\langle b_{2}^{L, S}(\theta ; \lambda), E a^{\prime}\right\rangle\right| \leq\left\|b_{2}^{L, S}(\theta ; \lambda)\right\| \cdot\left\|E a^{\prime}\right\| \sqrt{c_{22}(\theta ; \lambda)} \cdot\left\|a^{\prime}\right\|,
$$

so that

$$
\begin{gathered}
K_{\epsilon}^{L, S}(\theta ; \lambda) \leq \frac{\sqrt{c_{22}^{L, S}(\theta ; \lambda)}}{2 \epsilon} \int_{\frac{\epsilon}{\sqrt{c_{11}^{L, S}(\theta ; \lambda)}}}^{\frac{\epsilon}{\sqrt{c_{11}^{L, S}(\theta ; \lambda)}}} d a_{1}^{\prime} \int_{\mathbb{R}^{N-1}}\left\|a^{\prime}\right\| e^{-\frac{1}{4}\left\|a^{\prime}\right\|^{2}} \cdot e^{-\frac{1}{4}\left\|a^{\prime}\right\|^{2}} \frac{d a_{2}^{\prime} \ldots d a_{N}^{\prime}}{(2 \pi)^{N / 2}} \\
\leq C_{1} \sqrt{\frac{c_{22}^{L, S}(\theta ; \lambda)}{c_{11}^{L, S}(\theta ; \lambda)}},
\end{gathered}
$$

for some constant $C_{1}>0$, since $x e^{-\frac{1}{4} x^{2}}$ is bounded. Thus we have

$$
K_{\epsilon}^{L, S}(\theta ; \lambda) \leq C_{1} \sqrt{\frac{c_{22}^{L, S}(\theta ; \lambda)}{c_{11}^{L, S}(\theta ; \lambda)}} \leq C_{2} \lambda,
$$

for some constant $C_{2}>0$, where the last estimate in (65) follows from the asymptotics for the $c_{i j}$ 's in section 4 (in the long range case) and section 5 (in the short range case). Thus by (61) and the dominated convergence theorem,

$$
\mathbb{E} \tilde{\mathcal{I}}^{L, S}(\lambda)=\int_{0}^{\ell} K^{L, S}(\theta ; \lambda) d \theta
$$

where

$$
K^{L, S}(\theta ; \lambda):=\lim _{\epsilon \rightarrow 0^{+}} K_{\epsilon}^{L, S}(\theta ; \lambda) .
$$

The fundamental theorem of the calculus and (64) then imply that

$$
\begin{aligned}
K^{L, S}(\theta ; \lambda) & =\frac{1}{2 \pi \sqrt{\operatorname{det} C^{L, S}(\theta ; \lambda)}} \int_{-\infty}^{\infty}\left|v_{2}\right| \exp \left(-\frac{1}{2}\left(0, v_{2}\right) C^{L, S}(\theta ; \lambda)^{-1}\left(0, v_{2}\right)^{t}\right) d v_{2} \\
& =\frac{1}{2 \pi \sqrt{\operatorname{det} C^{L, S}(\theta ; \lambda)}} \int_{-\infty}^{\infty}\left|v_{2}\right| \exp \left(-\frac{1}{2} \frac{c_{11}^{L, S}(\theta ; \lambda)}{\operatorname{det} C^{L, S}(\theta ; \lambda)} v_{2}^{2}\right) d v_{2} \\
& =\frac{1}{2 \pi} \frac{\sqrt{\operatorname{det} C^{L, S}(\theta ; \lambda)}}{c_{11}^{L, S}(\theta ; \lambda)} \int_{-\infty}^{\infty}|z| \exp \left(-\frac{1}{2} z^{2}\right) d z \\
& =\frac{1}{\pi} \frac{\sqrt{c_{11}^{L, S}(\theta ; \lambda) c_{22}^{L, S}(\theta ; \lambda)-c_{12}^{L, S}(\theta ; \lambda)^{2}}}{c_{11}^{L, S}(\theta ; \lambda)}
\end{aligned}
$$


since $\int_{\mathbb{R}} \exp \left(-\frac{1}{2} z^{2}\right)|z| d z=2$.

\section{REFERENCES}

[A] Alexander J.W. A proof and extension of the Jordan-Brouwer separation theorem, Trans. Amer. Math. Soc. 23 (1922), 333-349.

[Be] Bérard, P. Volume des ensembles nodaux des fonctions propres du laplacien. BonySjoestrand-Meyer seminar, 1984-1985, Exp. No. 14 , 10 pp., École Polytech., Palaiseau, 1985.

[BG] Brüning, J; Gromes, D. Uber die Lnge der Knotenlinien schwingender Membranen, Math. Z. 1241972 79-82.

[BGS] Blum, G; Gnutzmann, S; Smilansky, U. Nodal Domains Statistics: A Criterion for Quantum Chaos. Phys. Rev. Lett. 88 (2002), 114101.

[BR] Berry, M. V. Statistics of nodal lines and points in chaotic quantum billiards: perimeter corrections, fluctuations, curvature J.Phys.A 35 (2002), 3025-3038.

[BSZ1] Bleher, Pavel; Shiffman, Bernard; Zelditch, Steve Universality and scaling of correlations between zeros on complex manifolds. Invent. Math. 142 (2000), no. 2, 351-395.

[BSZ2] Bleher, Pavel; Shiffman, Bernard; Zelditch, Steve Universality and scaling of zeros on symplectic manifolds. Random matrix models and their applications, 31-69, Math. Sci. Res. Inst. Publ., 40, Cambridge Univ. Press, Cambridge, 2001.

[CL] Cramer, H; Leadbetter, M. R. Stationary and related stochastic processes. Sample function properties and their applications. Reprint of the 1967 original. Dover Publications, Inc., Mineola, NY, 2004.

[DF] Donnelly, H; Fefferman, C. Nodal sets of eigenfunctions on Riemannian manifolds, Invent. Math. 93 (1988), 161-183.

[DF2] Donnelly, H; Fefferman, C. Nodal sets of eigenfunctions: Riemannian manifolds with boundary, Analysis, et cetera, 251-262, Academic Press, Boston, MA, 1990.

[DS] Dimassi, M; Sjoestrand, J. Spectral asymptotics in the semi-classical limit. London Mathematical Society Lecture Note Series, 268. Cambridge University Press, Cambridge, 1999.

[GW] Granville, A.; Wigman, I. The distribution of the zeroes of random stationary trigonometric polynomials, available online http://arxiv.org/abs/0809.1848.

[HZ] Hassell, A; Zelditch, S. Quantum ergodicity of boundary values of eigenfunctions, Comm. Math. Phys. 248, 119-168, 2004.

[I] Ivrii, V. The second term of the spectral asymptotics for a Laplace-Beltrami operator on manifolds with boundary. (Russian) Funktsional. Anal. i Prilozhen. 14 (1980), no. $2,25-34$

[M1] Melrose, R. The trace of the wave group. Microlocal analysis (Boulder, Colo., 1983), 127167, Contemp. Math., 27, Amer. Math. Soc., Providence, RI, 1984.

[MSj] Melrose, R.; Sjoestrand J. Singularities of boundary value problems. Comm. Pure Appl. Math., 31 (1978), 593-617.

[NS] Nazarov, F.; Sodin M., On the Number of Nodal Domains of Random Spherical Harmonics (arXiv:0706.2409).

[Oz] Ozawa. Asymptotic property of eigenfunction of the Laplacian at the boundary. Osaka J. Math. 30 (1993), 303314.

[RW] Rudnick, Z.; Wigman, I. On the volume of nodal sets for eigenfunctions of the Laplacian on the torus. Ann. Henri Poincare 9 (2008), no. 1, 109-130.

[S] Seeley, R. The resolvent of an elliptic boundary problem, Amer. J. Math. 91 (1969), 889920.

[SZ] Shiffman, B.; Zelditch, S. Distribution of zeros of random and quantum chaotic sections of positive line bundles. Comm. Math. Phys. 200 (1999), no. 3, 661-683.

[TZ1] Toth, J.A.; Zelditch, S. Counting nodal lines which touch the boundary of an analytic domain, J, Diff. Geom. 81 (2009), no. 3, 649-686.

[U] Uhlenbeck, K. Generic properties of eigenfunctions. Amer. J. Math. 98 (1976), no. 4, 1059-1078.

[W] Wigman, I. On the distribution of the nodal sets of random spherical harmonics, available online http://aps.arxiv.org/abs/0805.2768 
[Y1] Yau, S.T. Survey on partial differential equations in differential geometry. Seminar on Differential Geometry, pp. 3-71, Ann. of Math. Stud., 102, Princeton Univ. Press, Princeton, N.J., 1982.

[Y2] Yau, S.T. Open problems in geometry. Differential geometry: partial differential equations on manifolds (Los Angeles, CA, 1990), 1-28, Proc. Sympos. Pure Math., 54, Part 1, Amer. Math. Soc., Providence, RI, 1993.

[Z1] Zelditch, S. Billiards and boundary traces of eigenfunctions, Proceedings of the Forges-Les-Eaux Conference on PDE (2003).

[Z2] Zelditch, S. Real and complex zeros of Riemannian random waves, arXiv:0803.4334 (to appear in Contemp. Math. AMS Proceedings of the Conference "Spectral Analysis in Geometry and Number Theory on the occasion of Toshikazu Sunada's 60th birthday").

Department of Mathematics and Statistics, McGill University, 805 SherBrooke Str. West, Montréal QC H3A 2K6, Canada.

E-mail address: jtoth@math.mcgill.ca

Centre de recherches mathématiques (CRM), Université de Montréal C.P. 6128, sucC. Centre-ville Montréal, QuéBec H3C 3J7, CANAdA

E-mail address: wigman@crm.umontreal.ca 